\title{
Stochastic semiclassical gravity
}

\author{
Rosario Martín and Enric Verdaguer* \\ Departament de Física Fonamental, Universitat de Barcelona, Avenida Diagonal 647, E-08028 Barcelona, Spain
}

(Received 7 April 1999; published 17 September 1999)

\begin{abstract}
In the first part of this paper, we show that the semiclassical Einstein-Langevin equation, introduced in the framework of a stochastic generalization of semiclassical gravity to describe the back reaction of matter stress-energy fluctuations, can be formally derived from a functional method based on the influence functional of Feynman and Vernon. In the second part, we derive a number of results for background solutions of semiclassical gravity consisting of stationary and conformally stationary spacetimes and scalar fields in thermal equilibrium states. For these cases, fluctuation-dissipation relations are derived. We also show that particle creation is related to the vacuum stress-energy fluctuations and that it is enhanced by the presence of stochastic metric fluctuations. [S0556-2821(99)02318-8]
\end{abstract}

PACS number(s): 04.62.+v, 05.40.-a

\section{INTRODUCTION}

It is generally believed that there must be a regime in which the gravitational field can be treated as a classical or "quasiclassical", field, but its interaction with quantum matter fields cannot be neglected. The standard approach to describe such a regime is the semiclassical theory of gravity based on the semiclassical Einstein equation. This is a generalization of the Einstein equation for a classical metric when the expectation value of the stress-energy tensor of quantum matter fields is the source of curvature. The semiclassical theory of gravity is mathematically consistent and fairly well understood, at least for linear matter fields [1-5].

One expects that semiclassical gravity could be derived as an approximation of a fundamental quantum theory of gravity. However, in the absence of such a fundamental theory, the scope and limits of the semiclassical theory are less well understood $[1,6]$. It has been pointed out, nevertheless, that this semiclassical theory may not be valid when the matter fields have important quantum stress-energy fluctuations $[1,2,4,7]$. When this is the case, the stress-energy fluctuations may have relevant back-reaction effects on the spacetime geometry in the form of induced gravitational fluctuations [7]. A number of examples have been studied, both in cosmological and in flat spacetimes, where, for some states of the matter fields, the stress-energy tensor has significant fluctuations [8]. It is thus necessary to extend the semiclassical theory of gravity to determine the effect of such fluctuations.

To address this problem, different approaches have been adopted. The aim of the first part of the present paper is to unify two of these approaches.

One of these approaches relies on the idea, first proposed by $\mathrm{Hu}$ [9] in the context of semiclassical cosmology, of viewing the metric field as the "system" of interest and the matter fields as being part of its "environment." This approach leads naturally to the influence functional formalism of Feynman and Vernon [10]. In this formalism, the integration of the environment variables in a path integral yields the influence functional, from which one can define an effective

\footnotetext{
*Also at Institut de Física d'Altes Energies (IFAE).
}

action for the dynamics of the system [11-18]. This approach has been extensively used in the literature, not only in the framework of semiclassical cosmology [12-14,19-25], but also in the context of analogous semiclassical regimes in quantum mechanics $[15,17,26]$ and in quantum field theory $[18,27-30]$. It is based on the observation that the semiclassical equation can be directly derived from the effective action of Feynman and Vernon [12,18,19,22,23,29]. When computing this effective action perturbatively up to quadratic order in its variables, one usually finds some imaginary terms which do not contribute to the semiclassical equation. The key point is then to formally identify the contribution of such terms in the influence functional with the characteristic functional of a Gaussian stochastic source. Assuming that, in the semiclassical regime, this stochastic source interacts with the system variables, equations of the Langevin type can be derived for these variables. However, since this approach relies on a purely formal identification, doubts may be raised on the physical significance of the derived equations.

An alternative approach has been introduced in a recent paper [31]. In that work, we proposed a stochastic semiclassical theory of gravity as a perturbative generalization of semiclassical gravity to describe the back reaction of the lowest order stress-energy fluctuations. The idea is in fact quite simple. One starts realizing that, for a given solution of semiclassical gravity, the lowest order matter stress-energy fluctuations can be associated to a classical stochastic tensor field. Then, we seek an equation which incorporates in a consistent way this stochastic tensor as the source of linear perturbations to the semiclassical metric. The resulting equation is the semiclassical Einstein-Langevin equation.

We should emphasize that, even if the metric fluctuations in this theory are classical (stochastic fluctuations), their origin is presumably quantum. This is so not only because these metric fluctuations are induced by the fluctuations of a quantum operator, but also because they are supposed to describe some remnants of the quantum gravity fluctuations after some mechanism for decoherence and classicalization of the metric field [32-36]. From the formal assumption that such a mechanism is the Gell-Mann and Hartle mechanism of environment-induced decoherence of suitably coarse-grained system variables [32,33], one may, in fact, derive the stochastic semiclassical theory [37]. Nevertheless, that deriva- 
tion is of course formal, given that, due to the lack of the full quantum theory of gravity, the classicalization mechanism for the gravitational field is not understood. One expects that the stochastic semiclassical theory is valid when the characteristic time and space scales of variation of the metric field are well above its characteristic decoherence scales. In this regime, the theory can be applied to compute correlation functions of gravitational perturbations for points separated by scales larger than these decoherence scales. Hence, this theory may have a number of interesting applications in black hole physics and in cosmology, particularly in view of the problem of structure formation. Some examples of simple applications have already been given in Refs. $[22,25,31]$.

The purpose of the second part of the paper is to derive some general results concerning stochastic semiclassical gravity for stationary and conformally stationary background solutions of semiclassical gravity (for conformal matter fields in the latter case). We analyze two issues: the existence of a fluctuation-dissipation relation and the creation of particles by stochastic metric perturbations.

Under very general conditions, a fluctuation-dissipation relation is known to exist in models of quantum mechanics, and also in some models of quantum many-body systems or quantum fields in the presence of classical fields [38$44,42,45]$. This is a relation between quantum fluctuations of a system in a state of thermal equilibrium and the dissipative properties of this system caused by classical linear perturbations on it. The idea of a fluctuation-dissipation relation in the theory of quantum fields in curved spacetimes and in the semiclassical back-reaction problem was already present in some early papers $[46,47,9]$. A fluctuation-dissipation relation has been found in some of the previous derivations of semiclassical Langevin-type equations [14,19,23,24]. Some authors believe that such a relation should always be present and embody the physics of the back reaction of matter fields on the gravitational field $[14,23,24,48,49]$. It is also believed that noise and dissipation must be related to the creation of particles by stochastic metric perturbations $[9,12-$ $14,21,22,48,49]$.

In stationary and conformally stationary spacetimes (for conformal fields in the latter case), one can define a state of thermal equilibrium for the matter fields. When the background solution of semiclassical gravity is of one of these types, we can identify a dissipation kernel in the corresponding semiclassical Einstein-Langevin equation which is related to the fluctuations of the stochastic source by a fluctuation-dissipation relation. We also study the production of particles by stochastic metric perturbations to such backgrounds: we relate particle creation to the vacuum stressenergy fluctuations and we show that the mean value of created particles is enhanced by the presence of metric fluctuations.

The plan of the paper is the following. In Sec. II, we construct the stochastic semiclassical theory of gravity to describe the back reaction of the stress-energy fluctuations on the spacetime. In Sec. III, we show that the semiclassical Einstein-Langevin equation obtained in Sec. II can actually be formally derived with the functional approach. This con- nection clarifies the physical meaning of the Langevin-type equations previously derived by functional methods [1214,19-25], since it shows that the formally introduced stochastic source is directly related to the matter stress-energy fluctuations. We then use the functional approach to write the Einstein-Langevin equation in an explicit form, which is more suitable for specific calculations. In Sec. IV, we derive the fluctuation-dissipation relation for stationary and conformally stationary backgrounds and the results for particle creation by stochastic metric perturbations. Finally, in Sec. V, we summarize our main conclusions.

Throughout this paper we use the $(+++)$ sign conventions and the abstract index notation of Ref. [50], and we work with units in which $c=\hbar=1$.

\section{STOCHASTIC SEMICLASSICAL GRAVITY}

In this section, we construct the stochastic semiclassical theory of gravity as a perturbative extension of semiclassical gravity to describe the back reaction of quantum stressenergy fluctuations on the gravitational field. Let us begin with a brief overview of the semiclassical theory of gravity interacting with linear matter fields. Let $\left(\mathcal{M}, g_{a b}\right)$ be a globally hyperbolic four-dimensional spacetime and consider a linear quantum field $\Phi$ on it. For the sake of definiteness, we will take $\Phi$ as a real scalar field, but all the analysis of this section is valid for any kind of linear quantum field or for a set of linear independent quantum fields. Throughout this section we shall work in the Heisenberg picture. The field operator in this picture, $\hat{\Phi}$, is an operator-valued distribution solution of the Klein-Gordon equation,

$$
\left(\square-m^{2}-\xi R\right) \hat{\Phi}=0,
$$

where $m$ is the mass, $\square \equiv \nabla_{a} \nabla^{a}$, with $\nabla_{a}$ being the covariant derivative associated to the metric $g_{a b}$, and $\xi$ is a dimensionless parameter coupling the field to the scalar curvature $R$. To indicate that the field operator is a functional of the metric $g_{a b}$, we will write $\hat{\Phi}[g](x)$.

The classical stress-energy tensor is obtained by functional derivation of the classical action for the field in a background spacetime $\left(\mathcal{M}, g_{a b}\right)$ with respect to the metric. This tensor is a functional $T_{a b}[g, \Phi]$ of the metric $g_{a b}$ and of the classical field $\Phi$. For a real scalar field, it is

$$
\begin{aligned}
T_{a b}[g, \Phi]= & \nabla_{a} \Phi \nabla_{b} \Phi-\frac{1}{2} g_{a b} \nabla^{c} \Phi \nabla_{c} \Phi-\frac{1}{2} g_{a b} m^{2} \Phi^{2} \\
& +\xi\left(g_{a b} \square-\nabla_{a} \nabla_{b}+G_{a b}\right) \Phi^{2}
\end{aligned}
$$

where $G_{a b}$ is the Einstein tensor. The next step is to define a stress-energy tensor operator $\hat{T}_{a b}[g](x)$. In a naive way, one would replace the classical field $\Phi$ in the functional $T_{a b}[g, \Phi]$ by its corresponding quantum operator $\hat{\Phi}[g]$. However, since the field operator is well-defined only as a distribution on spacetime and this procedure involves taking the product of two distributions at the same spacetime point, the formal expression for $\hat{T}_{a b}[g]$ is ill-defined and we need a 
regularization procedure. We may formally think of a regularized "operator" $\hat{T}_{a b}[g](x ; \Omega)$, depending on some regulator $\Omega$, defined by giving a precise prescription for computing its matrix elements for physically acceptable states of the field. These states are assumed to be Hadamard states on the Fock space of a Hadamard vacuum state [1]. The states may have to be regularized also in some way and the procedure may involve some analytic continuation in the values of the regulator. Of course, if we remove the regularization in the results for these matrix elements, we would obtain infinite quantities.

Once the regularization prescription has been introduced, a renormalized and regularized stress-energy "operator" $\hat{T}_{a b}^{R}[g](x ; \Omega)$ may be defined as

$$
\hat{T}_{a b}^{R}[g](x ; \Omega)=\hat{T}_{a b}[g](x ; \Omega)+F_{a b}^{C}[g](x ; \Omega) \hat{I},
$$

where $\hat{I}$ is the identity operator and $F_{a b}^{C}[g]$ are some symmetric tensor counterterms, which can be written in terms of the regulator $\Omega$ and local functionals of the metric $g_{c d}(x) .{ }^{1}$ These counterterms can and must be chosen in such a way that, for any pair of physically acceptable states $|\psi\rangle$ and $|\varphi\rangle$, the matrix element of the renormalized operator $\hat{T}_{a b}^{R}[g]$, defined by

$$
\left\langle\psi\left|\hat{T}_{a b}^{R}\right| \varphi\right\rangle \equiv \lim _{\Omega \rightarrow \Omega_{p}}\left\langle\psi\left|\hat{T}_{a b}^{R}\right| \varphi\right\rangle(\Omega),
$$

where $\Omega_{p}$ means the "physical value" of the regulator, is finite (well defined as a distribution) and satisfies Wald's axioms $[3,2]$. Using the point-splitting or the dimensional regularization methods, these counterterms can be extracted from the singular part of a Schwinger-DeWitt series $[3,51,52]$. The choice of these counterterms is not unique, each different choice is called a "renormalization scheme," and this leads to some ambiguity in the definition of the renormalized stress-energy tensor operator. But this ambiguity can be absorbed into the renormalized coupling constants appearing in the equations of motion for the gravitational field. Thus, the ambiguity is only a mathematical artifact of the separation of the action into a gravitational part and a matter part, but the physically relevant equations are in fact unique $[3,53]$.

The semiclassical Einstein equation for the metric $g_{a b}$ can then be written as

\footnotetext{
${ }^{1}$ In the point-splitting regularization method, for instance, one introduces a point $y$ in a normal neighborhood of the point $x$, so some non-local dependence on the metric is explicitly introduced in the regularized stress-energy operator and then also in the counterterms. Using this regularization technique, the regulator can be taken as the vector $\sigma^{a}(x, y)$, which is the tangent vector at the point $x$ to the geodesic joining $x$ and $y$ with length equal to the arc length along this geodesic. In this case, the counterterms can be written in terms of the vector $\sigma^{a}(x, y)$ and tensors which are local functionals of the metric $g_{a b}(x)[3,51]$.
}

$$
\frac{1}{8 \pi G}\left(G_{a b}[g]+\Lambda g_{a b}\right)-2\left(\alpha A_{a b}+\beta B_{a b}\right)[g]=\left\langle\hat{T}_{a b}^{R}\right\rangle[g],
$$

where $\left\langle\hat{T}_{a b}^{R}\right\rangle[g]$ is the expectation value of $\hat{T}_{a b}^{R}[g]$ in some physically acceptable state of the quantum field on the spacetime $\left(\mathcal{M}, g_{a b}\right)$. The notation $\left\langle\hat{T}_{a b}^{R}\right\rangle[g]$ is used to indicate that this expectation value is a functional of the metric $g_{c d}$, not only because the stress-energy tensor operator depends on the metric, but also because the state of the matter field depends on the spacetime (in general, such a state depends on the global structure of the spacetime manifold). In Eq. (2.5), $G, \Lambda, \alpha$ and $\beta$ are renormalized coupling constants, respectively, the Newtonian gravitational constant, the cosmological constant and two dimensionless coupling constants. These constants may be seen as the result of "dressing" the bare coupling constants in a suitably regularized version of the gravitational part of the action,

$$
\begin{aligned}
S_{g}[g] \equiv & \int d^{4} x \sqrt{-g}\left[\frac{1}{16 \pi G_{B}}\left(R-2 \Lambda_{B}\right)\right. \\
& \left.+\alpha_{B} C_{a b c d} C^{a b c d}+\beta_{B} R^{2}\right],
\end{aligned}
$$

where $C_{a b c d}$ is the Weyl tensor and the subindex $B$ in the coupling constants means "bare." These renormalized coupling constants are supposed to be determined experimentally (for the specific renormalization scheme that one has chosen and for the characteristic scales of the physics under consideration). The tensors $A_{a b}$ and $B_{a b}$ in Eq. (2.5) come from the functional derivatives with respect to the metric of the terms quadratic in the curvature in $S_{g}[g]$, which are needed to ensure the renormalizability of the theory. These tensors are explicitly given by

$$
\begin{aligned}
A^{a b} \equiv & \frac{1}{\sqrt{-g}} \frac{\delta}{\delta g_{a b}} \int d^{4} x \sqrt{-g} C_{c d e f} C^{c d e f} \\
= & \frac{1}{2} g^{a b} C_{c d e f} C^{c d e f}-2 R^{a c d e} R_{c d e}^{b}+4 R^{a c} R_{c}^{b}-\frac{2}{3} R R^{a b} \\
& -2 \square R^{a b}+\frac{2}{3} \nabla^{a} \nabla^{b} R+\frac{1}{3} g^{a b} \square R
\end{aligned}
$$

and

$$
\begin{aligned}
B^{a b} & \equiv \frac{1}{\sqrt{-g}} \frac{\delta}{\delta g_{a b}} \int d^{4} x \sqrt{-g} R^{2} \\
& =\frac{1}{2} g^{a b} R^{2}-2 R R^{a b}+2 \nabla^{a} \nabla^{b} R-2 g^{a b} \square R,
\end{aligned}
$$

where $R_{a b c d}$ is the Riemann tensor and $R_{a b}$ is the Ricci tensor. Note that each of the terms in Eq. (2.5) has vanishing divergence. Notice also that we could add a classical stress- 
energy tensor to the right hand side of Eq. (2.5), if we had a classical matter source, but, for simplicity, we shall ignore such a term.

As long as the gravitational field is assumed to be described by a classical Lorentzian metric $g_{a b}$, the semiclassical Einstein equation seems to be the only physically plausible dynamical equation for this metric. The reason is that, in classical general relativity, the metric $g_{a b}$ couples to matter through the stress-energy tensor. For a field quantized on the spacetime $\left(\mathcal{M}, g_{a b}\right)$ and for a given state of this field, the expectation value of the renormalized stress-energy tensor operator is the only physically observable (up to the ambiguity mentioned above) c-number stress-energy tensor that we can construct.

A solution of semiclassical gravity consists of a spacetime $\left(\mathcal{M}, g_{a b}\right)$, a quantum field operator $\hat{\Phi}[g]$ satisfying Eq. (2.1), and a physically acceptable state $|\psi\rangle[g]$ for this field (which can also be a mixed state characterized by a density operator), such that Eq. (2.5) is satisfied when the expectation value in the state $|\psi\rangle[g]$ of the renormalized operator $\hat{T}_{a b}^{R}[g]$ is put on the right hand side.

Let us now introduce stress-energy fluctuations. Given a solution of semiclassical gravity, the stress-energy tensor will in general have quantum fluctuations. To lowest order, such fluctuations are described by the bi-tensor, which shall be called noise kernel, defined by

$$
8 N_{a b c d}(x, y) \equiv \lim _{\Omega \rightarrow \Omega_{p}}\left\langle\left\{\hat{t}_{a b}(x), \hat{t}_{c d}(y)\right\}\right\rangle[g](\Omega),
$$

where $\{$,$\} means the anticommutator and \hat{t}_{a b}(x ; \Omega)$ $\equiv \hat{T}_{a b}(x ; \Omega)-\left\langle\hat{T}_{a b}(x)\right\rangle(\Omega)$. Note that we have defined this noise kernel in terms of the unrenormalized "operator" $\hat{T}_{a b}[g](x ; \Omega)$. For a linear quantum field, this can be done because the ultraviolet singular behavior of $\left\langle\hat{T}_{a b}(x) \hat{T}_{c d}(y)\right\rangle(\Omega) \quad$ is the same as that of $\left\langle\hat{T}_{a b}(x)\right\rangle(\Omega)\left\langle\hat{T}_{c d}(y)\right\rangle(\Omega)$, so $N_{a b c d}(x, y)$ is free of ultraviolet divergencies. One can trivially see from the substitution of (2.3) into (2.9) that we can replace $\hat{T}_{a b}[g](x ; \Omega)$ by the renormalized operator $\hat{T}_{a b}^{R}[g](x)$, and omit the limit $\Omega$ $\rightarrow \Omega_{p}$, in the last expression. The result is obviously independent of the renormalization scheme that one chooses to define $\hat{T}_{a b}^{R}$.

As a perturbative correction to semiclassical gravity, we want now to introduce an equation in which the stress-energy fluctuations described by Eq. (2.9) are the source of classical gravitational fluctuations. Thus, we assume that the gravitational field is described by $g_{a b}+h_{a b}$, where $h_{a b}$ is a linear perturbation to the background metric $g_{a b}$, solution of Eq. (2.5). The renormalized stress-energy operator and the state of the quantum field may be denoted by $\hat{T}_{a b}^{R}[g+h]$ and $|\psi\rangle[g+h]$, respectively, and $\left\langle\hat{T}_{a b}^{R}\right\rangle[g+h]$ is the corresponding expectation value.

Let us introduce a Gaussian stochastic tensor field $\xi_{a b}$ defined by the following correlators:

$$
\left\langle\xi_{a b}(x)\right\rangle_{c}=0, \quad\left\langle\xi_{a b}(x) \xi_{c d}(y)\right\rangle_{c}=N_{a b c d}(x, y),
$$

where \langle\rangle$_{c}$ means statistical average. In general, the two-point correlation function of a stochastic tensor field $\xi_{a b}$ must be a symmetric, in the sense that $\left\langle\xi_{a b}(x) \xi_{c d}(y)\right\rangle_{c}$ $=\left\langle\xi_{c d}(y) \xi_{a b}(x)\right\rangle_{c}$, and positive semi-definite real bi-tensor field. Since the renormalized operator $\hat{T}_{a b}^{R}$ is self-adjoint, it is easy to see from the definition (2.9) that $N_{a b c d}(x, y)$ satisfies all these conditions. Therefore, the relations (2.10), with the cumulants of higher order taken to be zero, do truly characterize a stochastic tensor field $\xi_{a b}$. The simplest equation which can incorporate in a consistent way the stress-energy fluctuations described by $N_{a b c d}(x, y)$ as the source of classical metric fluctuations is

$$
\begin{aligned}
& \frac{1}{8 \pi G}\left(G_{a b}[g+h]+\Lambda\left(g_{a b}+h_{a b}\right)\right)-2\left(\alpha A_{a b}+\beta B_{a b}\right)[g+h] \\
& \quad=\left\langle\hat{T}_{a b}^{R}\right\rangle[g+h]+2 \xi_{a b},
\end{aligned}
$$

which must be understood as a dynamical equation for $h_{a b}$ to linear order. Equation (2.11) is the semiclassical EinsteinLangevin equation, which gives a first order correction to semiclassical gravity. One could also seek equations describing higher order corrections, which would involve higher order stress-energy fluctuations, but, for simplicity, we shall stick to the lowest order.

In order to check the consistency of Eq. (2.11), note that the term $\xi_{a b}$ does not depend on $h_{c d}$, since it is completely determined from the solution of semiclassical gravity by the correlators (2.10). Even so, this term must be considered as of first order in perturbation theory around semiclassical gravity. As shown in Ref. [31], $\xi_{a b}$ is covariantly conserved up to first order in this perturbation theory, in the sense that $\nabla^{a} \xi_{a b}$ behaves deterministically as the zero vector field on $\mathcal{M}$ ( $\nabla^{a}$ is the covariant derivative associated to the background metric $g_{a b}$ ). It is thus consistent to include the term $\xi_{a b}$ in the right hand side of Eq. (2.11).

It was also shown in Ref. [31] that for a conformal field, i.e., a field whose classical action is conformally invariant (e.g., a massless conformally coupled scalar field), $\xi_{a b}$ is "traceless" up to first order in perturbation theory, since $g^{a b} \xi_{a b}$ behaves deterministically as a vanishing scalar. Hence, in the case of a conformal matter field, the trace of the right hand side of Eq. (2.11) comes only from the trace anomaly.

Since Eq. (2.11) is a linear stochastic equation for $h_{a b}$ with an inhomogeneous term $\xi_{a b}$, a solution can be formally written as a functional $h_{a b}[\xi]$. Such a solution can be characterized by the whole family of its correlation functions. From the average of Eq. (2.11), the average of the metric, $g_{a b}+\left\langle h_{a b}\right\rangle_{c}$, must be a solution of the semiclassical Einstein equation linearized around $g_{a b}$. The fluctuations of the metric around this average can be described by the moments of order higher than one of the stochastic field $h_{a b}^{\mathrm{f}}[\xi]$ $\equiv h_{a b}[\xi]-\left\langle h_{a b}\right\rangle_{c}$.

Finally, for the solutions of Eq. (2.11) we have the gauge freedom $h_{a b} \rightarrow h_{a b}^{\prime} \equiv h_{a b}+\nabla_{a} \zeta_{b}+\nabla_{b} \zeta_{a}$, where $\zeta^{a}$ is any stochastic vector field on $\mathcal{M}$ which is a functional of $\xi_{c d}$, and $\zeta_{a} \equiv g_{a b} \zeta^{b}$. Note that the tensors which appear in Eq. (2.11) transform as $R_{a b}\left[g+h^{\prime}\right]=R_{a b}[g+h]+£_{\zeta} R_{a b}[g]$ (to linear 
order in the perturbations), where $£_{\zeta}$ is the Lie derivative with respect to $\zeta^{a}$. If we substitute $h_{a b}$ by $h_{a b}^{\prime}$ in Eq. (2.11), we get Eq. (2.11) plus the Lie derivative of a combination of the tensors which appear in Eq. (2.5). This last tensorial combination vanishes when Eq. (2.5) is satisfied. Thus, it is necessary that the set $\left(\mathcal{M}, g_{a b}, \hat{\Phi}[g],|\psi\rangle[g]\right)$ be a solution of semiclassical gravity to ensure that the Einstein-Langevin equation (2.11) is gauge invariant.

\section{DERIVATION FROM AN INFLUENCE ACTION}

The purpose of this section is to derive the semiclassical Einstein-Langevin equation (2.11) by a method based on functional techniques. The same method has been in fact used in the literature to derive Langevin-type equations in the context of semiclassical cosmology [12-14,19-25] and of analogous semiclassical regimes for systems of quantum mechanics $[15,17,26]$ and of quantum field theory $[18,27-$ 30]. Using these functional techniques, we also work out the Einstein-Langevin equation more explicitly, in a form more suitable for specific calculations. Here, we consider again the simplest case of a linear real scalar field $\Phi$.

These functional techniques are based on the closed time path (CTP) functional formalism, due to Schwinger and Keldysh $[41,54]$. This formalism is designed to obtain expectation values of field operators in a direct way and it is suited to derive dynamical equations for expectation values; see Refs. [55,56,23] for detailed reviews. In our case, this formalism will be useful to obtain an expression for the expectation value $\left\langle\hat{T}^{a b}\right\rangle[g+h]$ as an expansion in the metric perturbation. When the full quantum system consists of a distinguished subsystem (the "system" of interest) interacting with an environment (the remaining degrees of freedom), the CTP functional formalism turns out to be related $[12,18,19,23,28,29,37]$ to the influence functional formalism of Feynman and Vernon [10]. In this latter formalism, the integration of the environment variables in a CTP path integral yields the influence functional, from which one can define an effective action for the dynamics of the system [1118]. Applying this influence functional formalism to our problem, the semiclassical Einstein-Langevin equation will be formally derived in Sec. III B.

In our case, we consider the metric field $g_{a b}(x)$ as the "system" degrees of freedom, and the scalar field $\Phi(x)$ and also some "high-momentum" gravitational modes [36] as the "environment" variables. Unfortunately, since the form of a complete quantum theory of gravity interacting with matter is unknown, we do not know what these "highmomentum" gravitational modes are. Such a fundamental quantum theory might not even be a field theory, in which case the metric and scalar fields would not be fundamental objects [48]. Thus, in this case, we cannot attempt to evaluate the influence action of Feynman and Vernon starting from the fundamental quantum theory and performing the path integrations in the environment variables. Instead, we introduce the influence action for an effective quantum field theory of gravity and matter $[57,13]$, in which such "highmomentum" gravitational modes are assumed to have been already "integrated out." Adopting the usual procedure of effective field theories [58,57], one has to take the effective action for the metric and the scalar field of the most general local form compatible with general covariance: $S[g, \Phi]$ $\equiv S_{g}[g]+S_{m}[g, \Phi]+\cdots$, where $S_{g}[g]$ is given by Eq. (2.6),

$$
S_{m}[g, \Phi] \equiv-\frac{1}{2} \int d^{4} x \sqrt{-g}\left[g^{a b} \partial_{a} \Phi \partial_{b} \Phi+\left(m^{2}+\xi R\right) \Phi^{2}\right],
$$

and the dots stand for terms of order higher than two in the curvature and in the number of derivatives of the scalar field [because of the Gauss-Bonnet theorem in four spacetime dimensions, no further terms of second order in the curvature are needed in the gravitational action (2.6)]. In this paper, we shall neglect the higher order terms as well as self-interaction terms for the scalar field. The second order terms are necessary to renormalize one-loop ultraviolet divergencies of the scalar field stress tensor. Since $\mathcal{M}$ is a globally hyperbolic manifold, we can foliate it by a family of $t=$ constant Cauchy hypersurfaces $\Sigma_{t}$. We denote by $\mathbf{x}$ the coordinates on each of these hypersurfaces, and by $t_{i}$ and $t_{f}$ some initial and final times, respectively. The integration domain for the action terms must be understood as a compact region $\mathcal{U}$ of the manifold $\mathcal{M}$, bounded by the hypersurfaces $\Sigma_{t_{i}}$ and $\Sigma_{t_{f}}$.

Assuming the form (3.1) for the effective action which couples the scalar and the metric fields, we can now introduce the corresponding influence functional. This is a functional of two copies of the metric field that we denote by $g_{a b}^{+}$ and $g_{a b}^{-}$. Let us assume that, in the quantum effective theory, the state of the full system (the scalar and the metric fields) in the Schrödinger picture at the initial time $t=t_{i}$ can be described by a factorizable density operator, i.e., a density operator which can be written as the tensor product of two operators on the Hilbert spaces of the metric and of the scalar field. Let $\hat{\rho}^{\mathrm{S}}\left(t_{i}\right)$ be the density operator describing the initial state of the scalar field. If we consider the theory of a scalar field quantized in the classical background spacetime $\left(\mathcal{M}, g_{a b}\right)$ through the action (3.1), a state in the Heisenberg picture described by a density operator $\hat{\rho}[g]$ corresponds to this state. Let $\left\{|\varphi(\mathbf{x})\rangle^{\mathrm{S}}\right\}$ be the basis of eigenstates of the scalar field operator $\hat{\Phi}^{\mathrm{S}}(\mathbf{x})$ in the Schrödinger picture: $\hat{\Phi}^{\mathrm{S}}(\mathbf{x})|\varphi\rangle^{\mathrm{S}}=\varphi(\mathbf{x})|\varphi\rangle^{\mathrm{S}}$. The matrix elements of $\hat{\rho}^{\mathrm{S}}\left(t_{i}\right)$ in this basis will be written as $\rho_{i}[\varphi, \tilde{\varphi}] \equiv \mathrm{S}\left\langle\varphi\left|\hat{\rho}^{\mathrm{S}}\left(t_{i}\right)\right| \tilde{\varphi}\right\rangle^{\mathrm{S}}$. We can now introduce the influence functional as the following path integral over two copies of the scalar field:

$$
\mathcal{F}_{\mathrm{IF}}\left[g^{+}, g^{-}\right] \equiv \int \mathcal{D}\left[\Phi_{+}\right] \mathcal{D}\left[\Phi_{-}\right] \rho_{i}\left[\Phi_{+}\left(t_{i}\right), \Phi_{-}\left(t_{i}\right)\right] \delta\left[\Phi_{+}\left(t_{f}\right)-\Phi_{-}\left(t_{f}\right)\right] e^{i\left(S_{m}\left[g^{+}, \Phi_{+}\right]-S_{m}\left[g^{-}, \Phi_{-}\right]\right)}
$$


The above double path integral can be rewritten as a closed time path (CTP) integral, namely, as an integral over a single copy of field paths with two different time branches, one going forward in time from $t_{i}$ to $t_{f}$, and the other going backward in time from $t_{f}$ to $t_{i}$. From this influence functional, the influence action, $S_{\mathrm{IF}}\left[g^{+}, g^{-}\right]$, and the effective action of Feynman and Vernon, $S_{\text {eff }}\left[g^{+}, g^{-}\right]$, are defined by $\mathcal{F}_{\mathrm{IF}}\left[g^{+}, g^{-}\right] \equiv e^{i S_{\mathrm{IF}}\left[g^{+}, g^{-}\right]} \quad$ and $S_{\mathrm{eff}}\left[g^{+}, g^{-}\right] \equiv S_{g}\left[g^{+}\right]$ $-S_{g}\left[g^{-}\right]+S_{\mathrm{IF}}\left[g^{+}, g^{-}\right]$.

Expression (3.2) is ill-defined; it must be regularized to get a meaningful influence functional. We shall assume that we can use dimensional regularization, that is, that we can give sense to Eq. (3.2) by dimensional continuation of all the quantities that appear in this expression. We should point out, nevertheless, that for this regularization method to work one must be able to perform an analytic continuation to Riemmanian signature [59]. Thus, we substitute the action $S_{m}$ in Eq. (3.2) by some generalization to $n$ spacetime dimensions, which may be chosen as

$$
\begin{aligned}
S_{m}\left[g, \Phi_{n}\right]= & -\frac{1}{2} \int d^{n} x \sqrt{-g}\left[g^{a b} \partial_{a} \Phi_{n} \partial_{b} \Phi_{n}\right. \\
& \left.+\left(m^{2}+\xi R\right) \Phi_{n}^{2}\right],
\end{aligned}
$$

where we use a notation in which a subindex $n$ is attached to these quantities that have different physical dimensions than the corresponding physical quantities in four dimensions. A quantity with the subindex $n$ can always be associated to another without this subindex by means of a mass scale $\mu$; thus, for the scalar field $\Phi_{n}=\mu^{(n-4) / 2} \Phi$.

We also need to substitute the action (2.6) by some suitable generalization to $n$ spacetime dimensions. We take

$$
\begin{aligned}
S_{g}[g]= & \mu^{n-4} \int d^{n} x \sqrt{-g}\left[\frac{1}{16 \pi G_{B}}\left(R-2 \Lambda_{B}\right)\right. \\
& \left.+\frac{2}{3} \alpha_{B}\left(R_{a b c d} R^{a b c d}-R_{a b} R^{a b}\right)+\beta_{B} R^{2}\right] .
\end{aligned}
$$

By the Gauss-Bonnet theorem, this action gives for $n=4$ the same equations of motion as the action (2.6). The form of Eq. (3.4) is suggested by the Schwinger-DeWitt analysis of the ultraviolet divergencies in the matter stress-energy tensor using dimensional regularization [52]. Using Eqs. (3.3) and (3.4), one can write the effective action of Feynman and Vernon, $S_{\text {eff }}\left[g^{+}, g^{-}\right]$, in dimensional regularization. Since the action terms (3.3) and (3.4) contain second order derivatives of the metric, one should also add some boundary terms $[50,13]$. The effect of these terms is to cancel out the boundary terms which appear when taking variations of $S_{\text {efft }}\left[g^{+}, g^{-}\right]$keeping the value of $g_{a b}^{+}$and $g_{a b}^{-}$fixed on the boundary of $\mathcal{U}$. Alternatively, in order to obtain the equations of motion for the metric in the semiclassical regime, we can work with the action terms (3.3) and (3.4) (without boundary terms) and neglect all boundary terms when taking variations with respect to $g_{a b}^{ \pm}$. From now on, all the functional derivatives with respect to the metric will be understood in this sense.

\section{A. The semiclassical Einstein equation in dimensional regularization}

From the action (3.3), we can define the stress-energy tensor functional in the usual way,

$$
T^{a b}\left[g, \Phi_{n}\right](x) \equiv \frac{2}{\sqrt{-g(x)}} \frac{\delta S_{m}\left[g, \Phi_{n}\right]}{\delta g_{a b}(x)},
$$

which yields Eq. (2.2). Working in the Heisenberg picture, we can now formally introduce the regularized stress-energy tensor operator as

$$
\hat{T}_{n}^{a b}[g] \equiv T^{a b}\left[g, \hat{\Phi}_{n}[g]\right], \quad \hat{T}^{a b}[g] \equiv \mu^{-(n-4)} \hat{T}_{n}^{a b}[g],
$$

where $\hat{\Phi}_{n}[g](x)$ is the field operator, which satisfies the Klein-Gordon equation (2.1) in $n$ spacetime dimensions, and where we use a symmetrical ordering (Weyl ordering) prescription for the operators. Using the Klein-Gordon equation, the stress-energy operator can be written as

$$
\hat{T}_{n}^{a b}[g]=\frac{1}{2}\left\{\nabla^{a} \hat{\Phi}_{n}[g], \nabla^{b} \hat{\Phi}_{n}[g]\right\}+\mathcal{D}^{a b}[g] \hat{\Phi}_{n}^{2}[g],
$$

where $\mathcal{D}^{a b}[g]$ is the differential operator

$$
\mathcal{D}_{x}^{a b} \equiv\left(\xi-\frac{1}{4}\right) g^{a b}(x) \square_{x}+\xi\left(R^{a b}(x)-\nabla_{x}^{a} \nabla_{x}^{b}\right) .
$$

From the definitions (3.2), (3.5) and (3.6), one can see that

$$
\left\langle\hat{T}_{n}^{a b}(x)\right\rangle[g]=\left.\frac{2}{\sqrt{-g(x)}} \frac{\delta S_{\mathrm{IF}}\left[g^{+}, g^{-}\right]}{\delta g_{a b}^{+}(x)}\right|_{g^{+}=g^{-}=g},
$$

where the expectation value is taken in the $n$-dimensional spacetime generalization of the state described by $\hat{\rho}[g]$. Therefore, differentiating $S_{\text {eff }}\left[g^{+}, g^{-}\right]=S_{g}\left[g^{+}\right]-S_{g}\left[g^{-}\right]$ $+S_{\mathrm{IF}}\left[g^{+}, g^{-}\right]$with respect to $g_{a b}^{+}$, and then setting $g_{a b}^{+}$ $=g_{a b}^{-}=g_{a b}$, we get the semiclassical Einstein equation in dimensional regularization:

$$
\begin{aligned}
& \frac{1}{8 \pi G_{B}}\left(G^{a b}[g]+\Lambda_{B} g^{a b}\right)-\left(\frac{4}{3} \alpha_{B} D^{a b}+2 \beta_{B} B^{a b}\right)[g] \\
& \quad=\mu^{-(n-4)}\left\langle\hat{T}_{n}^{a b}\right\rangle[g],
\end{aligned}
$$

where 


$$
\begin{aligned}
D^{a b} \equiv & \frac{1}{\sqrt{-g}} \frac{\delta}{\delta g_{a b}} \int d^{n} x \sqrt{-g}\left(R_{c d e f} R^{c d e f}-R_{c d} R^{c d}\right) \\
= & \frac{1}{2} g^{a b}\left(R_{c d e f} R^{c d e f}-R_{c d} R^{c d}+\square R\right) \\
& -2 R^{a c d e} R_{c d e}^{b}-2 R^{a c b d} R_{c d}+4 R^{a c} R_{c}^{b} \\
& -3 \square R^{a b}+\nabla^{a} \nabla^{b} R
\end{aligned}
$$

and $B^{a b}$ is defined as in Eq. (2.8) but for $n$ spacetime dimensions, although its explicit expression in terms of the metric and curvature tensors is the same. When $n=4$, one has that $D^{a b}=(3 / 2) A^{a b}$, where $A^{a b}$ is the tensor defined in Eq. (2.7). From Eq. (3.10), renormalizing the coupling constants to eliminate the "divergencies" in $\mu^{-(n-4)}\left\langle\hat{T}_{n}^{a b}\right\rangle[g]$, and taking the limit $n \rightarrow 4$, we get the physical semiclassical Einstein equation (2.5).

\section{B. A formal derivation of the semiclassical Einstein-Langevin equation}

In the spirit of the previous section, we now seek a dynamical equation for a linear perturbation $h_{a b}$ to a semiclassical metric $g_{a b}$, solution of Eq. (3.10) in $n$ spacetime dimensions. From the result of the previous subsection, if such an equation were simply a linearized semiclassical Einstein equation, it could be obtained from an expansion of the effective action $S_{\text {eff }}\left[g+h^{+}, g+h^{-}\right]$. In particular, since, from Eq. (3.9), we have that

$$
\left\langle\hat{T}_{n}^{a b}(x)\right\rangle[g+h]=\left.\frac{2}{\sqrt{-\operatorname{det}(g+h)(x)}} \frac{\delta S_{\mathrm{IF}}\left[g+h^{+}, g+h^{-}\right]}{\delta h_{a b}^{+}(x)}\right|_{h^{+}=h^{-}=h},
$$

the expansion of $\left\langle\hat{T}_{n}^{a b}\right\rangle[g+h]$ to linear order in $h_{a b}$ can be obtained from an expansion of the influence action $S_{\mathrm{IF}}[g$ $\left.+h^{+}, g+h^{-}\right]$up to second order in $h_{a b}^{ \pm}$.

To perform the expansion of the influence action, we have to compute the first and second order functional derivatives of $S_{\mathrm{IF}}\left[g^{+}, g^{-}\right]$and then set $g_{a b}^{+}=g_{a b}^{-}=g_{a b}$. If we do so using the path integral representation (3.2), we can interpret these derivatives as expectation values of operators. The relevant second order derivatives are

$$
\begin{aligned}
& \left.\frac{1}{\sqrt{-g(x)} \sqrt{-g(y)}} \frac{\delta^{2} S_{\mathrm{IF}}\left[g^{+}, g^{-}\right]}{\delta g_{a b}^{+}(x) \delta g_{c d}^{+}(y)}\right|_{g^{+}=g^{-}=g}=-H_{\mathrm{S}_{n}}^{a b c d}[g](x, y)-K_{n}^{a b c d}[g](x, y)+i N_{n}^{a b c d}[g](x, y), \\
& \left.\frac{1}{\sqrt{-g(x)} \sqrt{-g(y)}} \frac{\delta^{2} S_{\mathrm{IF}}\left[g^{+}, g^{-}\right]}{\delta g_{a b}^{+}(x) \delta g_{c d}^{-}(y)}\right|_{g^{+}=g^{-}=g}=-H_{\mathrm{A}_{n}}^{a b c d}[g](x, y)-i N_{n}^{a b c d}[g](x, y),
\end{aligned}
$$

where

$$
\begin{aligned}
& N_{n}^{a b c d}[g](x, y) \equiv \frac{1}{8}\left\langle\left\{\hat{t}_{n}^{a b}(x), \hat{t}_{n}^{c d}(y)\right\}\right\rangle[g], \\
& H_{\mathrm{S}_{n}}^{a b c d}[g](x, y) \equiv \frac{1}{4} \operatorname{Im}\left\langle\mathrm{T}^{*}\left[\hat{T}_{n}^{a b}(x) \hat{T}_{n}^{c d}(y)\right]\right\rangle[g], \\
& H_{\mathrm{A}_{n}}^{a b c d}[g](x, y) \equiv-\frac{i}{8}\left\langle\left[\hat{T}_{n}^{a b}(x), \hat{T}_{n}^{c d}(y)\right]\right\rangle[g], \\
& K_{n}^{a b c d}[g](x, y) \equiv \frac{-1}{\sqrt{-g(x)} \sqrt{-g(y)}}\left\langle\left.\frac{\delta^{2} S_{m}\left[g, \Phi_{n}\right]}{\delta g_{a b}(x) \delta g_{c d}(y)}\right|_{\Phi_{n}=\hat{\Phi}_{n}}\right\rangle[g],
\end{aligned}
$$

with $\hat{t}_{n}^{a b} \equiv \hat{T}_{n}^{a b}-\left\langle\hat{T}_{n}^{a b}\right\rangle$, and using again a Weyl ordering prescription for the operators in the last of these expressions. Here, [ , ] means the commutator, and we use the symbol T* to denote that, first, we have to time order the field operators $\hat{\Phi}_{n}$ and then apply the derivative operators which appear in each term of the product $T^{a b}(x) T^{c d}(y)$, where $T^{a b}$ is the functional (2.2). For instance,

$$
\mathrm{T}^{*}\left(\nabla_{x}^{a} \hat{\Phi}_{n}(x) \nabla_{x}^{b} \hat{\Phi}_{n}(x) \nabla_{y}^{c} \hat{\Phi}_{n}(y) \nabla_{y}^{d} \hat{\Phi}_{n}(y)\right)=\lim _{\substack{x_{1}, x_{2} \rightarrow x \\ x_{3}, x_{4} \rightarrow y}} \nabla_{x_{1}}^{a} \nabla_{x_{2}}^{b} \nabla_{x_{3}}^{c} \nabla_{x_{4}}^{d} \mathrm{~T}\left(\hat{\Phi}_{n}\left(x_{1}\right) \hat{\Phi}_{n}\left(x_{2}\right) \hat{\Phi}_{n}\left(x_{3}\right) \hat{\Phi}_{n}\left(x_{4}\right)\right),
$$


where $\mathrm{T}$ is the usual time ordering. This $\mathrm{T}^{*}$ "time ordering" arises because we have path integrals containing products of derivatives of the field, which can be expressed as derivatives of the path integrals which do not contain such derivatives. Notice, from the definitions (3.14), that all the kernels which appear in expressions (3.13) are real and that $H_{\mathrm{A}_{n}}^{a b c d}$ is also free of ultraviolet divergencies in the limit $n \rightarrow 4$.

From Eqs. (3.13) and (3.14), it is clear that the imaginary part of the influence action, which does not contribute to the semiclassical Einstein equation (3.10) because the expectation value of $\hat{T}_{n}^{a b}[g]$ is real, contains information on the fluctuations of this operator. From Eqs. (3.9) and (3.13), taking into account that $S_{\mathrm{IF}}[g, g]=0$ and that $S_{\mathrm{IF}}\left[g^{-}, g^{+}\right]$ $=-S_{\mathrm{IF}}^{*}\left[g^{+}, g^{-}\right]$, we can write the expansion for the influ- ence action $S_{\mathrm{IF}}\left[g+h^{+}, g+h^{-}\right]$around a background metric $g_{a b}$ in terms of the kernels (3.14). Taking into account that these kernels satisfy the symmetry relations

$$
\begin{aligned}
& H_{\mathrm{S}_{n}}^{a b c d}(x, y)=H_{\mathrm{S}_{n}}^{c d a b}(y, x), \\
& H_{\mathrm{A}_{n}}^{a b c d}(x, y)=-H_{\mathrm{A}_{n}}^{c d a b}(y, x), \\
& K_{n}^{a b c d}(x, y)=K_{n}^{c d a b}(y, x),
\end{aligned}
$$

and introducing a new kernel

$$
H_{n}^{a b c d}(x, y) \equiv H_{\mathrm{S}_{n}}^{a b c d}(x, y)+H_{\mathrm{A}_{n}}^{a b c d}(x, y),
$$

this expansion can be finally written as

$$
\begin{aligned}
S_{\mathrm{IF}}\left[g+h^{+}, g+h^{-}\right]= & \frac{1}{2} \int d^{n} x \sqrt{-g(x)}\left\langle\hat{T}_{n}^{a b}(x)\right\rangle[g]\left[h_{a b}(x)\right]-\frac{1}{2} \int d^{n} x d^{n} y \sqrt{-g(x)} \sqrt{-g(y)}\left[h_{a b}(x)\right]\left(H_{n}^{a b c d}[g](x, y)\right. \\
& \left.+K_{n}^{a b c d}[g](x, y)\right)\left\{h_{c d}(y)\right\}+\frac{i}{2} \int d^{n} x d^{n} y \sqrt{-g(x)} \sqrt{-g(y)}\left[h_{a b}(x)\right] N_{n}^{a b c d}[g](x, y)\left[h_{c d}(y)\right]+0\left(h^{3}\right),
\end{aligned}
$$

where we have used the notation

$$
\left[h_{a b}\right] \equiv h_{a b}^{+}-h_{a b}^{-}, \quad\left\{h_{a b}\right\} \equiv h_{a b}^{+}+h_{a b}^{-} .
$$

We are now in the position to carry out the formal derivation of the semiclassical Einstein-Langevin equation. The procedure is well known [12-14,19-25,15,17,26,18,27-30]; it consists of deriving a new "improved" effective action using the the following identity:

$$
e^{-(1 / 2) \int d^{n} x d^{n} y \sqrt{-g(x)} \sqrt{-g(y)}\left[h_{a b}(x)\right] N_{n}^{a b c d}(x, y)\left[h_{c d}(y)\right]}=\int \mathcal{D}\left[\xi_{n}\right] \mathcal{P}\left[\xi_{n}\right] e^{i \int d^{n} x \sqrt{-g(x)} \xi_{n}^{a b}(x)\left[h_{a b}(x)\right]},
$$

where $\mathcal{P}\left[\xi_{n}\right]$ is the probability distribution functional of a Gaussian stochastic tensor $\xi_{n}^{a b}$ characterized by the correlators

$$
\left\langle\xi_{n}^{a b}(x)\right\rangle_{c}=0, \quad\left\langle\xi_{n}^{a b}(x) \xi_{n}^{c d}(y)\right\rangle_{c}=N_{n}^{a b c d}[g](x, y),
$$

with $N_{n}^{a b c d}$ given in Eq. (3.14), and where the path integration measure is assumed to be a scalar under diffeomor- phisms of $\left(\mathcal{M}, g_{a b}\right)$. The above identity follows from the identification of the right hand side of Eq. (3.20) with the characteristic functional for the stochastic field $\xi_{n}^{a b}$. In fact, by differentiation of this expression with respect to $\left[h_{a b}\right]$, it can be checked that this is the characteristic functional of a stochastic field characterized by the correlators (3.21). When $N_{n}^{a b c d}(x, y)$ is strictly positive definite, the probability distribution functional for $\xi_{n}^{a b}$ is explicitly given by

$$
\mathcal{P}\left[\xi_{n}\right]=\frac{e^{-(1 / 2) \int d^{n} x d^{n} y \sqrt{-g(x)} \sqrt{-g(y)} \xi_{n}^{a b}(x) N_{n a b c d}^{-1}(x, y) \xi_{n}^{c d}(y)}}{\int \mathcal{D}\left[\bar{\xi}_{n}\right] e^{-(1 / 2) \int d^{n} z d^{n} w \sqrt{-g(z)} \sqrt{-g(w)} \bar{\xi}_{n}^{e f}(z) N_{n e f g h}^{-1}(z, w) \bar{\xi}_{n}^{g h}(w)}},
$$

where $N_{n a b c d}^{-1}[g](x, y)$ is the inverse of $N_{n}^{a b c d}[g](x, y)$ defined by 


$$
\int d^{n} z \sqrt{-g(z)} N_{n}^{a b e f}(x, z) N_{n e f c d}^{-1}(z, y)=\frac{1}{2}\left(\delta_{c}^{a} \delta_{d}^{b}+\delta_{d}^{a} \delta_{c}^{b}\right) \frac{\delta^{n}(x-y)}{\sqrt{-g(x)}}
$$

Using the identity (3.20), we can write the modulus of the influence functional in the approximation (3.18) as

$$
\begin{aligned}
\left|\mathcal{F}_{\mathrm{IF}}\left[g+h^{+}, g+h^{-}\right]\right| & =e^{-\operatorname{Im} S_{\mathrm{IF}}\left[g+h^{+}, g+h^{-}\right]} \\
& =\left\langle e^{i \int d^{n} x \sqrt{-g(x)} \xi_{n}^{a b}(x)\left[h_{a b}(x)\right]}\right\rangle_{c}
\end{aligned}
$$

where \langle\rangle$_{c}$ means statistical average over the stochastic tensor $\xi_{n}^{a b}$. Thus, the effect of the imaginary part of the influence action (3.18) on the corresponding influence functional is equivalent to the averaged effect of the stochastic source $\xi_{n}^{a b}$ coupled linearly to the perturbations $h_{a b}^{ \pm}$. The influence functional, in the approximation (3.18), can be written as a statistical average over $\xi_{n}^{a b}$ :

$$
\mathcal{F}_{\mathrm{IF}}\left[g+h^{+}, g+h^{-}\right]=\left\langle e^{i \mathcal{A}_{\mathrm{IF}}^{\mathrm{eff}}\left[h^{+}, h^{-} ; g ; \xi_{n}\right]}\right\rangle_{c}
$$

with

$$
\begin{aligned}
\mathcal{A}_{\mathrm{IF}}^{\mathrm{eff}}\left[h^{+}, h^{-} ; g ; \xi_{n}\right] \equiv & \operatorname{Re} S_{\mathrm{IF}}\left[g+h^{+}, g+h^{-}\right] \\
& +\int d^{n} x \sqrt{-g(x)} \xi_{n}^{a b}(x)\left[h_{a b}(x)\right] \\
& +0\left(h^{3}\right)
\end{aligned}
$$

where $\operatorname{Re} S_{\text {IF }}$ can be read from the expansion (3.18). Note that the stochastic term in this action contains the information of the imaginary part of $S_{\mathrm{IF}}$. Introducing a new "improved" effective action

$$
\begin{aligned}
\mathcal{A}_{\mathrm{eff}}\left[h^{+}, h^{-} ; g ; \xi_{n}\right] \equiv & S_{g}\left[g+h^{+}\right]-S_{g}\left[g+h^{-}\right] \\
& +\mathcal{A}_{\mathrm{IF}}^{\mathrm{eff}}\left[h^{+}, h^{-} ; g ; \xi_{n}\right],
\end{aligned}
$$

where $S_{g}\left[g+h^{ \pm}\right]$has to be expanded up to second order in the perturbations $h_{a b}^{ \pm}$, the equation of motion for $h_{a b}$ can be derived as

$$
\left.\frac{1}{\sqrt{-\operatorname{det}(g+h)(x)}} \frac{\delta \mathcal{A}_{\mathrm{eff}}\left[h^{+}, h^{-} ; g ; \xi_{n}\right]}{\delta h_{a b}^{+}(x)}\right|_{h^{+}=h^{-}=h}=0
$$

From Eq. (3.12), taking into account that only the real part of the influence action contributes to the expectation value of the stress-energy tensor, we get, to linear order in $h_{a b}$,

$$
\frac{1}{8 \pi G_{B}}\left(G^{a b}[g+h]+\Lambda_{B}\left(g^{a b}-h^{a b}\right)\right)-\left(\frac{4}{3} \alpha_{B} D^{a b}+2 \beta_{B} B^{a b}\right)[g+h]=\mu^{-(n-4)}\left\langle\hat{T}_{n}^{a b}\right\rangle[g+h]+2 \mu^{-(n-4)} \xi_{n}^{a b},
$$

where $h^{a b} \equiv g^{a c} g^{b d} h_{c d}$, that is, $g^{a b}-h^{a b}+0\left(h^{2}\right)$ is the inverse of the metric $g_{a b}+h_{a b}$. This last equation is the semiclassical Einstein-Langevin equation in dimensional regularization. As we have pointed out in Sec. II, the two-point correlation function of the stochastic source in this equation [see Eq. (3.21)], given by the noise kernel defined in Eq. (3.14), is free of ultraviolet divergencies in the limit $n \rightarrow 4$. Therefore, in the Einstein-Langevin equation (3.29), one can perform exactly the same renormalization procedure as for the semiclassical Einstein equation (3.10). After this, Eq. (3.29) will yield the physical semiclassical EinsteinLangevin equation (2.11). The derivation presented in this paper clarifies the physical meaning of the stochastic source formally introduced in the effective action (3.26) by the identification (3.24), since it links its two-point correlation function to the stress-energy fluctuations by Eqs. (3.21) and (3.14).

There is also a connection between the equations obtained by this formal functional method and the equations derived from the (in general, also formal) assumption that decoherence and classicalization of suitably coarse-grained system variables is achieved through the mechanism proposed by Gell-Mann and Hartle [32] in the consistent histories formulation of a quantum theory. This last approach allows one to evaluate the probability distribution associated to such decoherent variables, given by the diagonal elements of a decoherence functional, and, under some approximations, to derive effective quasiclassical equations of motion for them. These effective equations of motion can be shown to coin- 
cide [37] with the semiclassical equations for the background and the Langevin-type equations for perturbations obtained from the above functional method. Taking this connection into account, we can also conclude that, if one formally assumes that the Gell-Mann and Hartle mechanism works for the metric field, one is led to the semiclassical Einstein equation and the semiclassical Einstein-Langevin equation for the background metric and for the metric perturbations, respectively [37].

We end this subsection with some comments on the relation between the semiclassical Einstein-Langevin equation (2.11) and the Langevin-type equations for stochastic metric perturbations recently derived in the literature [12-14,19$25]$. In these previous derivations, one starts with the influence functional (3.2), with the state of the scalar field assumed to be an "in' vacuum or an "in'" thermal state, and computes explicitly the expansion for the corresponding influence action around a specific metric background. One then applies the above formal method to derive a Langevin equation for the perturbations to this background. However, most of these derivations start with a "mini-superspace" model and, thus, the metric perturbations are assumed from the beginning to have a restrictive form. In those cases, the derived Langevin equations do not correspond exactly to our equation, Eq. (2.11), but to a "reduced" version of this equation, in which only some components of the noise kernel in Eq. (2.10) (or some particular combinations of them) influence the dynamics of the metric perturbations. Only those equations which have been derived starting from a completely general form for the metric perturbations $[19,20,23,24]$ are actually particular cases of the semiclassical EinsteinLangevin equation (2.11). Note, however, that the stochastic equations derived in Refs. [23,24] do not correspond exactly to Eq. (2.11), since the background (Minkowski spacetime and a scalar field in a thermal state) is not a solution of semiclassical gravity. In this case, for the reasons explained in Sec. II, the equation for the metric perturbations is not gauge invariant.

\section{Explicit linear form of the Einstein-Langevin equation}

We can write Eq. (3.29) in a more explicit form by working out the expansion of $\left\langle\hat{T}_{n}^{a b}\right\rangle[g+h]$ up to linear order in the perturbation $h_{a b}$. From Eq. (3.12), we see that this expansion can be easily obtained from Eq. (3.18). Noting, from Eq. (3.14), that

$$
\begin{aligned}
& K_{n}^{a b c d}[g](x, y) \\
&=-\frac{1}{4}\left\langle\hat{T}_{n}^{a b}(x)\right\rangle[g] \frac{g^{c d}(x)}{\sqrt{-g(y)}} \delta^{n}(x-y) \\
&-\frac{1}{2} \frac{1}{\sqrt{-g(y)}}\left\langle\left.\frac{\delta T^{a b}\left[g, \Phi_{n}\right](x)}{\delta g_{c d}(y)}\right|_{\Phi_{n}=\hat{\Phi}_{n}}\right\rangle[g],
\end{aligned}
$$

we get

$$
\left\langle\hat{T}_{n}^{a b}(x)\right\rangle[g+h]=\left\langle\hat{T}_{n}^{a b}(x)\right\rangle[g]+\left\langle\hat{T}_{n}^{(1) a b}[g ; h](x)\right\rangle[g]-2 \int d^{n} y \sqrt{-g(y)} H_{n}^{a b c d}[g](x, y) h_{c d}(y)+0\left(h^{2}\right),
$$

where the operator $\hat{T}_{n}^{(1) a b}$ is defined from the term of first order in the expansion of $T^{a b}\left[g+h, \Phi_{n}\right]$ as

$$
\begin{aligned}
T^{a b}\left[g+h, \Phi_{n}\right] & =T^{a b}\left[g, \Phi_{n}\right]+T^{(1) a b}\left[g, \Phi_{n} ; h\right]+0\left(h^{2}\right), \\
\hat{T}_{n}^{(1) a b}[g ; h] & \equiv T^{(1) a b}\left[g, \hat{\Phi}_{n}[g] ; h\right],
\end{aligned}
$$

using, as always, a Weyl ordering prescription for the opera- tors in the last definition. Note that the third term on the right hand side of Eq. (3.31) is a consequence of the dependence on $h_{c d}$ of the field operator $\hat{\Phi}_{n}[g+h]$ and of the density operator $\hat{\rho}[g+h]$.

Substituting Eq. (3.31) into Eq. (3.29), and taking into account that $g_{a b}$ satisfies the semiclassical Einstein equation (3.10), we can write the Einstein-Langevin equation (3.29) as

$$
\begin{aligned}
& \frac{1}{8 \pi G_{B}}\left(G^{(1) a b}[g ; h](x)-\Lambda_{B} h^{a b}(x)\right)-\frac{4}{3} \alpha_{B} D^{(1) a b}[g ; h](x)-2 \beta_{B} B^{(1) a b}[g ; h](x) \\
& \quad-\mu^{-(n-4)}\left\langle\hat{T}_{n}^{(1) a b}[g ; h](x)\right\rangle[g]+2 \int d^{n} y \sqrt{-g(y)} \mu^{-(n-4)} H_{n}^{a b c d}[g](x, y) h_{c d}(y)=2 \mu^{-(n-4)} \xi_{n}^{a b}(x) .
\end{aligned}
$$


In the last equation we have used the superindex (1) to denote the terms of first order in the expansion in $h_{a b}$ of the tensors $G^{a b}[g+h], D^{a b}[g+h]$ and $B^{a b}[g+h]$. Thus, for instance, $G^{a b}[g+h]=G^{a b}[g]+G^{(1) a b}[g ; h]+0\left(h^{2}\right)$. The explicit expressions for the tensors $G^{(1) a b}[g ; h], D^{(1) a b}[g ; h]$ and $B^{(1) a b}[g ; h]$ can be found in the Appendix of Ref. [37], and $T^{(1) a b}\left[g, \Phi_{n} ; h\right]$ is given in Appendix A. From $T^{(1) a b}\left[g, \Phi_{n} ; h\right]$, we can write an explicit expression for the operator $\hat{T}_{n}^{(1) a b}$. In fact, using the Klein-Gordon equation, and expressions (3.7) and (3.8) for the stress-energy operator, we have

$$
\begin{aligned}
\hat{T}_{n}^{(1) a b}[g ; h]= & \left(\frac{1}{2} g^{a b} h_{c d}-\delta_{c}^{a} h_{d}^{b}-\delta_{c}^{b} h_{d}^{a}\right) \hat{T}_{n}^{c d}[g] \\
& +\mathcal{F}^{a b}[g ; h] \hat{\Phi}_{n}^{2}[g],
\end{aligned}
$$

where $\mathcal{F}^{a b}[g ; h]$ is the differential operator

$$
\begin{aligned}
\mathcal{F}^{a b} \equiv & \left(\xi-\frac{1}{4}\right)\left(h^{a b}-\frac{1}{2} g^{a b} h_{c}^{c}\right) \square+\frac{\xi}{2}\left[\nabla^{c} \nabla^{a} h_{c}^{b}+\nabla^{c} \nabla^{b} h_{c}^{a}-\square h^{a b}-\nabla^{a} \nabla^{b} h_{c}^{c}-g^{a b} \nabla^{c} \nabla^{d} h_{c d}\right. \\
& \left.+g^{a b} \square h_{c}^{c}+\left(\nabla^{a} h_{c}^{b}+\nabla^{b} h_{c}^{a}-\nabla_{c} h^{a b}-2 g^{a b} \nabla^{d} h_{c d}+g^{a b} \nabla_{c} h_{d}^{d}\right) \nabla^{c}-g^{a b} h_{c d} \nabla^{c} \nabla^{d}\right] .
\end{aligned}
$$

It is understood that indices are raised with the background inverse metric $g^{a b}$ and that all the covariant derivatives are associated to the metric $g_{a b}$. Substituting expression (3.34) into Eq. (3.33), and using the semiclassical equation (3.10) to get an expression for $\mu^{-(n-4)}\left\langle\hat{T}_{n}^{a b}\right\rangle[g]$, we can finally write the semiclassical Einstein-Langevin equation in dimensional regularization as

$$
\begin{aligned}
& \frac{1}{8 \pi G_{B}}\left[G^{(1) a b}-\frac{1}{2} g^{a b} G^{c d} h_{c d}+G^{a c} h_{c}^{b}+G^{b c} h_{c}^{a}+\Lambda_{B}\left(h^{a b}-\frac{1}{2} g^{a b} h_{c}^{c}\right)\right](x) \\
& \quad-\frac{4}{3} \alpha_{B}\left(D^{(1) a b}-\frac{1}{2} g^{a b} D^{c d} h_{c d}+D^{a c} h_{c}^{b}+D^{b c} h_{c}^{a}\right)(x)-2 \beta_{B}\left(B^{(1) a b}-\frac{1}{2} g^{a b} B^{c d} h_{c d}+B^{a c} h_{c}^{b}+B^{b c} h_{c}^{a}\right)(x) \\
& \quad-\mu^{-(n-4)} \mathcal{F}_{x}^{a b}\left\langle\hat{\Phi}_{n}^{2}(x)\right\rangle[g]+2 \int d^{n} y \sqrt{-g(y)} \mu^{-(n-4)} H_{n}^{a b c d}[g](x, y) h_{c d}(y)=2 \mu^{-(n-4)} \xi_{n}^{a b}(x),
\end{aligned}
$$

where the tensors $G^{a b}, D^{a b}$ and $B^{a b}$ are computed from the semiclassical metric $g_{a b}$, and where we have omitted the functional dependence on $g_{a b}$ and $h_{a b}$ in $G^{(1) a b}, D^{(1) a b}$, $B^{(1) a b}$ and $\mathcal{F}^{a b}$ to simplify the notation. Notice that, in Eq. (3.36), all the ultraviolet divergencies in the limit $n \rightarrow 4$, which must be removed by renormalization of the coupling constants, are in $\left\langle\hat{\Phi}_{n}^{2}(x)\right\rangle$ and the symmetric part $H_{\mathrm{S}_{n}}^{a b c d}(x, y)$ of the kernel $H_{n}^{a b c d}(x, y)$, whereas the kernels $N_{n}^{a b c d}(x, y)$ and $H_{\mathrm{A}_{n}}^{a b c d}(x, y)$ are free of ultraviolet divergencies. These two last kernels can be written in terms of $F_{n}^{a b c d}[g](x, y)$ $\equiv\left\langle\hat{t}_{n}^{a b}(x) \hat{t}_{n}^{c d}(y)\right\rangle[g]$ as

$$
\begin{aligned}
& N_{n}^{a b c d}[g](x, y)=\frac{1}{4} \operatorname{Re} F_{n}^{a b c d}[g](x, y), \\
& H_{\mathrm{A}_{n}}^{a b c d}[g](x, y)=\frac{1}{4} \operatorname{Im} F_{n}^{a b c d}[g](x, y),
\end{aligned}
$$

where we have used that $2\left\langle\hat{t}_{n}^{a b}(x) \hat{t}_{n}^{c d}(y)\right\rangle$ $=\left\langle\left\{\hat{t}_{n}^{a b}(x), \hat{t}_{n}^{c d}(y)\right\}\right\rangle+\left\langle\left[\hat{t}_{n}^{a b}(x), \hat{t}_{n}^{c d}(y)\right]\right\rangle$, and the fact that the first term on the right hand side of this identity is real, whereas the second one is pure imaginary. Once we perform the renormalization procedure in Eq. (3.36), setting $n=4$ will yield the physical semiclassical Einstein-Langevin equation. Note that, due to the presence of the kernel $H_{n}^{a b c d}(x, y)$, this equation will be usually non-local in the metric perturbation.

\section{The kernels for a vacuum state}

We conclude this section by considering the case in which the expectation values that appear in the Einstein-Langevin equation (3.36) [see Eqs. (3.14)] are taken in a vacuum state $|0\rangle$ [for a field quantized on $\left(\mathcal{M}, g_{a b}\right)$ in the Heisenberg picture], such as, for instance, an "in" vacuum. In this case we can go further and write these expectation values in terms of the Wightman and Feynman functions, defined as

$$
\begin{gathered}
G_{n}^{+}(x, y) \equiv\left\langle 0\left|\hat{\Phi}_{n}(x) \hat{\Phi}_{n}(y)\right| 0\right\rangle[g], \\
i G_{F_{n}}(x, y) \equiv\left\langle 0\left|\mathrm{~T}\left(\hat{\Phi}_{n}(x) \hat{\Phi}_{n}(y)\right)\right| 0\right\rangle[g] .
\end{gathered}
$$

These expressions for the kernels in the Einstein-Langevin equation will be very useful for explicit calculations. To simplify the notation, we omit the functional dependence on the semiclassical metric $g_{a b}$, which will be understood in all the expressions below. 
From Eq. (3.37), we see that the kernels $N_{n}^{a b c d}(x, y)$ and $H_{\mathrm{A}_{n}}^{a b c d}(x, y)$ are the real and imaginary parts, respectively, of

$$
F_{n}^{a b c d}(x, y)=\left\langle 0\left|\hat{T}_{n}^{a b}(x) \hat{T}_{n}^{c d}(y)\right| 0\right\rangle-\left\langle 0\left|\hat{T}_{n}^{a b}(x)\right| 0\right\rangle\left\langle 0\left|\hat{T}_{n}^{c d}(y)\right| 0\right\rangle .
$$

Since, from Eq. (3.7), we can write the operator $\hat{T}_{n}^{a b}$ as a sum of terms of the form $\left\{\mathcal{A}_{x} \hat{\Phi}_{n}(x), \mathcal{B}_{x} \hat{\Phi}_{n}(x)\right\}$, where $\mathcal{A}_{x}$ and $\mathcal{B}_{x}$ are some differential operators, we can express $F_{n}^{a b c d}(x, y)$ in terms of the Wightman function using

$$
\begin{aligned}
& \left\langle\left\{\mathcal{A}_{x} \hat{\Phi}_{n}(x), \mathcal{B}_{x} \hat{\Phi}_{n}(x)\right\}\left\{\mathcal{C}_{y} \hat{\Phi}_{n}(y), \mathcal{D}_{y} \hat{\Phi}_{n}(y)\right\}\right\rangle-\left\langle\left\{\mathcal{A}_{x} \hat{\Phi}_{n}(x), \mathcal{B}_{x} \hat{\Phi}_{n}(x)\right\}\right\rangle\left\langle\left\{\mathcal{C}_{y} \hat{\Phi}_{n}(y), \mathcal{D}_{y} \hat{\Phi}_{n}(y)\right\}\right\rangle \\
& \quad=4 \mathcal{A}_{x} \mathcal{C}_{y} G_{n}^{+}(x, y) \mathcal{B}_{x} \mathcal{D}_{y} G_{n}^{+}(x, y)+4 \mathcal{A}_{x} \mathcal{D}_{y} G_{n}^{+}(x, y) \mathcal{B}_{x} \mathcal{C}_{y} G_{n}^{+}(x, y),
\end{aligned}
$$

where $\mathcal{C}_{x}$ and $\mathcal{D}_{x}$ are also some differential operators and where the expectation values are taken in the vacuum $|0\rangle$. This identity can be easily proved using Wick's theorem or by writing the operator $\hat{\Phi}_{n}(x)$ in terms of the creation and annihilation operators of the Fock representation corresponding to the vacuum $|0\rangle$. Using a Schwinger-DeWitt expansion for the Wightman function $G_{n}^{+}(x, y)$, one can actually see that the two terms on the right hand side of the last expression are free of ultraviolet divergencies in the limit $n \rightarrow 4$. Finally, we find

$$
\begin{aligned}
F_{n}^{a b c d}(x, y)= & \nabla_{x}^{a} \nabla_{y}^{c} G_{n}^{+}(x, y) \nabla_{x}^{b} \nabla_{y}^{d} G_{n}^{+}(x, y)+\nabla_{x}^{a} \nabla_{y}^{d} G_{n}^{+}(x, y) \nabla_{x}^{b} \nabla_{y}^{c} G_{n}^{+}(x, y) \\
& +2 \mathcal{D}_{x}^{a b}\left(\nabla_{y}^{c} G_{n}^{+}(x, y) \nabla_{y}^{d} G_{n}^{+}(x, y)\right)+2 \mathcal{D}_{y}^{c d}\left(\nabla_{x}^{a} G_{n}^{+}(x, y) \nabla_{x}^{b} G_{n}^{+}(x, y)\right)+2 \mathcal{D}_{x}^{a b} \mathcal{D}_{y}^{c d}\left(G_{n}^{+2}(x, y)\right)
\end{aligned}
$$

where $\mathcal{D}_{x}^{a b}$ is the differential operator (3.8). From this expression and the relations (3.37), we get expressions for the kernels $N_{n}^{a b c d}(x, y)$ and $H_{\mathrm{A}_{n}}^{a b c d}(x, y)$ in terms of the Wightman function $G_{n}^{+}(x, y)$.

The kernel $H_{\mathrm{S}_{n}}^{a b c d}(x, y)$, defined in Eq. (3.14), can be written in terms of the Feynman function noting that, from Wick's theorem,

$$
\operatorname{Im}\left\langle\mathrm{T}^{*}\left(\left\{\mathcal{A}_{x} \hat{\Phi}_{n}(x), \mathcal{B}_{x} \hat{\Phi}_{n}(x)\right\}\left\{\mathcal{C}_{y} \hat{\Phi}_{n}(y), \mathcal{D}_{y} \hat{\Phi}_{n}(y)\right\}\right)\right\rangle=-4 \operatorname{Im}\left[\mathcal{A}_{x} \mathcal{C}_{y} G_{F_{n}}(x, y) \mathcal{B}_{x} \mathcal{D}_{y} G_{F_{n}}(x, y)+\mathcal{A}_{x} \mathcal{D}_{y} G_{F_{n}}(x, y) \mathcal{B}_{x} \mathcal{C}_{y} G_{F_{n}}(x, y)\right]
$$

where, again, $\mathcal{A}_{x}, \mathcal{B}_{x}, \mathcal{C}_{x}$ and $\mathcal{D}_{x}$ are real differential operators and the expectation value is in the vacuum $|0\rangle$. The kernel $H_{\mathrm{S}_{n}}^{a b c d}(x, y)$ is then obtained by adding up the contribution of all the differential operators which appear in the product $T^{a b}(x) T^{c d}(y)$, where $T^{a b}$ is the functional (2.2). After a long calculation, we get

$$
\begin{aligned}
H_{\mathrm{S}_{n}}^{a b c d}(x, y)= & -\frac{1}{4} \operatorname{Im}\left[\nabla_{x}^{a} \nabla_{y}^{c} G_{F_{n}}(x, y) \nabla_{x}^{b} \nabla_{y}^{d} G_{F_{n}}(x, y)+\nabla_{x}^{a} \nabla_{y}^{d} G_{F_{n}}(x, y) \nabla_{x}^{b} \nabla_{y}^{c} G_{F_{n}}(x, y)\right. \\
& -g^{a b}(x) \nabla_{x}^{e} \nabla_{y}^{c} G_{F_{n}}(x, y) \nabla_{e}^{x} \nabla_{y}^{d} G_{F_{n}}(x, y)-g^{c d}(y) \nabla_{x}^{a} \nabla_{y}^{e} G_{F_{n}}(x, y) \nabla_{x}^{b} \nabla_{e}^{y} G_{F_{n}}(x, y) \\
& +\frac{1}{2} g^{a b}(x) g^{c d}(y) \nabla_{x}^{e} \nabla_{y}^{f} G_{F_{n}}(x, y) \nabla_{e}^{x} \nabla_{f}^{y} G_{F_{n}}(x, y)+\mathcal{K}_{x}^{a b}\left(2 \nabla_{y}^{c} G_{F_{n}}(x, y) \nabla_{y}^{d} G_{F_{n}}(x, y)\right. \\
& \left.-g^{c d}(y) \nabla_{y}^{e} G_{F_{n}}(x, y) \nabla_{e}^{y} G_{F_{n}}(x, y)\right)+\mathcal{K}_{y}^{c d}\left(2 \nabla_{x}^{a} G_{F_{n}}(x, y) \nabla_{x}^{b} G_{F_{n}}(x, y)\right. \\
& \left.\left.-g^{a b}(x) \nabla_{x}^{e} G_{F_{n}}(x, y) \nabla_{e}^{x} G_{F_{n}}(x, y)\right)+2 \mathcal{K}_{x}^{a b} \mathcal{K}_{y}^{c d}\left(G_{F_{n}}^{2}(x, y)\right)\right],
\end{aligned}
$$

where $\mathcal{K}_{x}^{a b}$ is the differential operator

$$
\mathcal{K}_{x}^{a b} \equiv \xi\left(g^{a b}(x) \square_{x}-\nabla_{x}^{a} \nabla_{x}^{b}+G^{a b}(x)\right)-\frac{1}{2} m^{2} g^{a b}(x) .
$$

An alternative expression for $H_{\mathrm{S}_{n}}^{a b c d}(x, y)$, which is more similar to expression (3.40), can be obtained taking into account that $G_{F_{n}}(x, y)$ is a Green function of the Klein-Gordon equation in $n$ spacetime dimensions, which satisfies

$$
\left(\square_{x}-m^{2}-\xi R(x)\right) G_{F_{n}}(x, y)=\frac{\delta^{n}(x-y)}{\sqrt{-g(x)}},
$$

and using that in dimensional regularization $\left[\delta^{n}(x-y)\right]^{2}$ $=0$. Finally, note that, in the vacuum $|0\rangle$, the term $\left\langle\hat{\Phi}_{n}^{2}(x)\right\rangle$ in Eq. (3.36) can also be written as $\left\langle\hat{\Phi}_{n}^{2}(x)\right\rangle=i G_{F_{n}}(x, x)$ $=G_{n}^{+}(x, x)$.

It is worth noting that, when the points $x$ and $y$ are space- 
like separated, $\hat{\Phi}_{n}(x)$ and $\hat{\Phi}_{n}(y)$ commute and, thus, $G_{n}^{+}(x, y)=i G_{F_{n}}(x, y)=(1 / 2)\left\langle 0\left|\left\{\hat{\Phi}_{n}(x), \hat{\Phi}_{n}(y)\right\}\right| 0\right\rangle$, which is real. Hence, from the above expressions, we have that $H_{\mathrm{A}_{n}}^{a b c d}(x, y)=H_{\mathrm{S}_{n}}^{a b c d}(x, y)=0$. This fact is not surprising since, from the causality of the expectation value of the stress-energy operator, we know that the non-local dependence on the metric perturbation in the Einstein-Langevin equation must be causal.

\section{FLUCTUATIONS IN STATIONARY AND CONFORMALLY STATIONARY BACKGROUNDS}

In this section, we derive a number of results concerning the stochastic semiclassical theory of gravity for two classes of background solutions of semiclassical gravity. The first class consists of a stationary spacetime and a scalar field in thermal equilibrium or in its vacuum state. In the second class, the spacetime is conformally stationary, the scalar field is massless and conformally coupled, and its state is the conformal vacuum or a thermal state built on the conformal vacuum. In Secs. IV A and IV B, we identify a kernel in the corresponding Einstein-Langevin equations which is related to the noise kernel by a fluctuation-dissipation relation. In Sec. IV C, we study the creation of particles by stochastic metric perturbations and see that this phenomenon can be related to the vacuum noise kernel. We show that the mean value of created particles is enhanced by the presence of metric fluctuations with respect to the same quantity in the "perturbed" semiclassical spacetime $\left(\mathcal{M}, g_{a b}+\left\langle h_{a b}\right\rangle_{c}\right)$.

Let us assume that the semiclassical spacetime $\left(\mathcal{M}, g_{a b}\right)$ is stationary, i.e., that it possesses a global timelike Killing vector field $\zeta^{a}, £_{\zeta} g_{a b}=0$, where $£_{\zeta}$ is the Lie derivative with respect to $\zeta^{a}$. Writing the Killing vector as $\zeta^{a}=(\partial / \partial t)^{a}$, this spacetime can be foliated by a family of Cauchy hypersurfaces $\Sigma_{t}$, labeled by the Killing time $t$, so we can give coordinates $(t, \mathbf{x})$ to each spacetime point, where $\mathbf{x}$ are the space coordinates on each of these hypersurfaces. Using this foliation, we can construct a Hamiltonian operator $\hat{H}[g]$ in the way described in Appendix B. This is a time independent, i.e., independent of the Cauchy hypersurface $\Sigma_{t}$, Hamiltonian operator, so it represents the Hamiltonian operator in both the Heisenberg and the Schrödinger pictures. In this case, there is a natural Fock representation based on a decomposition of the field operator $\hat{\Phi}_{n}[g]$ in a complete set of modes of positive frequencies $\omega_{k}$ with respect to $\zeta^{a}$, and their complex conjugates. ${ }^{2}$ This defines a natural Fock space, the many-particle states of which are eigenstates of the Hamiltonian $\hat{H}[g]$. Thus, the notion of particles is physically well defined in this spacetime $[1,60,5]$. The Hamiltonian operator in this Fock representation, renormalized by normal ordering, is given by $\hat{H}[g]=\sum_{k} \omega_{k} \hat{a}_{k}^{\dagger} \hat{a}_{k}$, where $\hat{a}_{k}^{\dagger}$ and $\hat{a}_{k}$ are

\footnotetext{
${ }^{2}$ In some cases, additional restrictions may be necessary to avoid infrared divergencies, such as that the scalar field is massive, $\mathrm{m}^{2}$ $\neq 0$, or that the norm of the Killing vector is not arbitrarily small $[1,60]$.
}

the creation and annihilation operators on the Fock space. Here, the summation must be understood as representing either a sum over a set of discrete indices or an integral with some suitable measure (or a combination of these two possibilities). The time-evolution operator corresponding to this Hamiltonian operator is then given by $\hat{\mathcal{U}}[g]\left(t, t^{\prime}\right)$ $\equiv \exp \left(-i \hat{H}[g]\left(t-t^{\prime}\right)\right)$.

In this section, even if we sometimes write $t_{i}$ or $t_{f}$, we shall always consider these initial and final times in the limit $t_{i} \rightarrow-\infty$ and $t_{f} \rightarrow+\infty$ (we assume that such limits can be taken).

\section{A. The fluctuation-dissipation relation in a stationary background}

For a real scalar field quantized on the stationary spacetime $\left(\mathcal{M}, g_{a b}\right)$, we can define a state of thermal equilibrium at temperature $T$. This state is described in the Heisenberg picture by the density operator of the grand canonical ensemble:

$$
\hat{\rho}[g]=\frac{e^{-\beta \hat{H}[g]}}{\operatorname{Tr}\left(e^{-\beta \hat{H}[g]}\right)},
$$

where $\beta \equiv 1 / k_{B} T$ and $k_{B}$ is Boltzmann's constant (there are no chemical potential terms because we deal with a real scalar field). This kind of thermal state for fields in stationary curved backgrounds was first considered in Refs. [61,62]. Since the density operator (4.1) commutes with the timeevolution operator $\hat{\mathcal{U}}[g]\left(t, t^{\prime}\right)$, the corresponding initial density operator in the Schrödinger picture is simply $\hat{\rho}^{\mathrm{S}}\left(t_{i}\right)$ $=\hat{\rho}[g]$.

Given any pair of operators in the Heisenberg picture, $\hat{P}[g](x)$ and $\hat{Q}[g](x)$, the expectation value $\left\langle\hat{P}(x) \hat{Q}\left(x^{\prime}\right)\right\rangle_{T}[g]$ depends on $t$ and $t^{\prime}$ only through the difference $t-t^{\prime}$, since

$$
\begin{aligned}
\left\langle\hat{P}(x) \hat{Q}\left(x^{\prime}\right)\right\rangle_{T}= & \operatorname{Tr}\left[\hat{\rho} \hat{P}(x) \hat{Q}\left(x^{\prime}\right)\right] \\
= & \operatorname{Tr}\left[\hat{\rho} \hat{P}^{\mathrm{S}}(\mathbf{x}) e^{-i \hat{H}\left(t-t^{\prime}\right)}\right. \\
& \left.\times \hat{Q}^{\mathrm{S}}\left(\mathbf{x}^{\prime}\right) e^{i \hat{H}\left(t-t^{\prime}\right)}\right],
\end{aligned}
$$

where $\hat{P}^{\mathrm{S}}(\mathbf{x})$ and $\hat{Q}^{\mathrm{S}}(\mathbf{x})$ are the operators in the Schrödinger picture corresponding to $\hat{P}(x)$ and $\hat{Q}(x)$, respectively, and we use \langle\rangle$_{T}$ to denote an expectation value in the state described by Eq. (4.1). In particular, with the choice $\hat{\rho}^{\mathrm{S}}\left(t_{i}\right)$ $=\hat{\rho}[g]$, the kernels $N_{n}^{a b c d}[g]\left(x, x^{\prime}\right), H_{\mathrm{S}_{n}}^{a b c d}[g]\left(x, x^{\prime}\right)$ and $H_{\mathrm{A}_{n}}^{a b c d}[g]\left(x, x^{\prime}\right)$ depend on the time coordinates as a function of $t-t^{\prime}$. Therefore, we can introduce Fourier transforms in the time coordinate as

$$
K\left(x, x^{\prime}\right)=\int_{-\infty}^{\infty} \frac{d \omega}{2 \pi} e^{-i \omega\left(t-t^{\prime}\right)} \tilde{K}\left(\omega ; \mathbf{x}, \mathbf{x}^{\prime}\right),
$$

where $K\left(x, x^{\prime}\right)$ is any function which depends on time only through $t-t^{\prime}$. 
As it is shown in Appendix B, Wick's theorem can be generalized for thermal $N$-point functions, defined as expectation values of products of the field operator in the state described by Eq. (4.1). It is then easy to see that the expressions found in Sec. III D also hold for the kernels $N_{n}^{a b c d}[g]\left(x, x^{\prime}\right), H_{\mathrm{S}_{n}}^{a b c d}[g]\left(x, x^{\prime}\right)$ and $H_{\mathrm{A}_{n}}^{a b c d}[g]\left(x, x^{\prime}\right)$ at finite $T$ if we replace the Wightman and Feynman functions (3.38) by the analogous thermal expectation values.

In this case, a simple relationship (in the form of a fluctuation-dissipation relation) exists between the kernels $N_{n}^{a b c d}[g]\left(x, x^{\prime}\right)$ and $H_{\mathrm{A}_{n}}^{a b c d}[g]\left(x, x^{\prime}\right)$. In fact, from Eq. (3.37), we can write these kernels as

$$
\begin{gathered}
8 N_{n}^{a b c d}\left(x, x^{\prime}\right)=F_{n}^{a b c d}\left(x, x^{\prime}\right)+F_{n}^{c d a b}\left(x^{\prime}, x\right), \\
8 i H_{\mathrm{A}_{n}}^{a b c d}\left(x, x^{\prime}\right)=F_{n}^{a b c d}\left(x, x^{\prime}\right)-F_{n}^{c d a b}\left(x^{\prime}, x\right),
\end{gathered}
$$

where we omit the functional dependence on $g_{a b}$. In terms of the Fourier transforms (4.3), these relations are

$$
\begin{aligned}
8 \widetilde{N}_{n}^{a b c d}\left(\omega ; \mathbf{x}, \mathbf{x}^{\prime}\right) & =\widetilde{F}_{n}^{a b c d}\left(\omega ; \mathbf{x}, \mathbf{x}^{\prime}\right)+\widetilde{F}_{n}^{c d a b}\left(-\omega ; \mathbf{x}^{\prime}, \mathbf{x}\right), \\
8 i \widetilde{H}_{\mathrm{A}_{n}}^{a b c d}\left(\omega ; \mathbf{x}, \mathbf{x}^{\prime}\right) & =\widetilde{F}_{n}^{a b c d}\left(\omega ; \mathbf{x}, \mathbf{x}^{\prime}\right)-\widetilde{F}_{n}^{c d a b}\left(-\omega ; \mathbf{x}^{\prime}, \mathbf{x}\right) .
\end{aligned}
$$

By analytically continuing $t$ to complex values in $F_{n}^{a b c d}\left(x, x^{\prime}\right)$, one can derive a symmetry relation for this bitensor which involves different values of this complex time. Taking into account that the time evolution of the operator $\hat{t}_{n}^{a b}$ is given in this stationary case by $\hat{t}_{n}^{a b}(t+\Delta t, \mathbf{x})$ $=e^{i \hat{H} \Delta t} \hat{t}_{n}^{a b}(t, \mathbf{x}) e^{-i \hat{H} \Delta t}$, and using the cyclic property of the trace, we get $F_{n}^{a b c d}\left(t, \mathbf{x} ; t^{\prime}, \mathbf{x}^{\prime}\right)=F_{n}^{c d a b}\left(t^{\prime}, \mathbf{x}^{\prime} ; t+i \beta, \mathbf{x}\right)$, or, equivalently, in terms of its Fourier transform,

$$
\widetilde{F}_{n}^{a b c d}\left(\omega ; \mathbf{x}, \mathbf{x}^{\prime}\right)=e^{\beta \omega} \widetilde{F}_{n}^{c d a b}\left(-\omega ; \mathbf{x}^{\prime}, \mathbf{x}\right) .
$$

This relation is known as the Kubo-Martin-Schwinger relation $[39,44]$. From this last expression and Eq. (4.5), we obtain the following simple relation between $\widetilde{N}_{n}^{a b c d}$ and $\widetilde{H}_{\mathrm{A}_{n}}^{a b c d}$ :

$$
\widetilde{H}_{\mathrm{A}_{n}}^{a b c d}\left(\omega ; \mathbf{x}, \mathbf{x}^{\prime}\right)=-i \tanh \left(\frac{\beta \omega}{2}\right) \widetilde{N}_{n}^{a b c d}\left(\omega ; \mathbf{x}, \mathbf{x}^{\prime}\right),
$$

which can also be written as

$$
H_{\mathrm{A}_{n}}^{a b c d}\left(t, \mathbf{x} ; t^{\prime}, \mathbf{x}^{\prime}\right)=\int_{-\infty}^{\infty} d t^{\prime \prime} K_{\mathrm{FD}}\left(t-t^{\prime \prime}\right), N_{n}^{a b c d}\left(t^{\prime \prime}, \mathbf{x} ; t^{\prime}, \mathbf{x}^{\prime}\right),
$$

with

$$
\begin{aligned}
K_{\mathrm{FD}}(t) & \equiv-\int_{0}^{\infty} \frac{d \omega}{\pi} \sin (\omega t) \tanh \left(\frac{\beta \omega}{2}\right) \\
& =-k_{B} T \mathrm{P}\left[\operatorname{csch}\left(\pi k_{B} T t\right)\right],
\end{aligned}
$$

where P denotes a Cauchy principal value distribution.
Since, as we have pointed out above, the kernels $H_{\mathrm{A}_{n}}^{\text {abcd }}$ and $N_{n}^{a b c d}$ are free of ultraviolet divergencies in the limit $n$ $\rightarrow 4$, we can define

$$
\begin{aligned}
& H_{\mathrm{A}}^{a b c d}\left(x, x^{\prime}\right) \equiv \lim _{n \rightarrow 4} \mu^{-2(n-4)} H_{\mathrm{A}_{n}}^{a b c d}\left(x, x^{\prime}\right), \\
& N^{a b c d}\left(x, x^{\prime}\right) \equiv \lim _{n \rightarrow 4} \mu^{-2(n-4)} N_{n}^{a b c d}\left(x, x^{\prime}\right),
\end{aligned}
$$

which are the kernels that appear in the physical semiclassical Einstein-Langevin equation, Eq. (2.11), after performing the renormalization procedure in Eq. (3.36). These physical kernels will also satisfy the relation (4.8) or, equivalently, their Fourier transforms will satisfy Eq. (4.7). These results are independent of the regularization method used.

The relation (4.7) can be written in an alternative way. Introducing a new kernel (this is actually a family of kernels) defined by $\widetilde{H}_{\mathrm{A}_{n}}^{a b c d}\left(\omega ; \mathbf{x}, \mathbf{x}^{\prime}\right) \equiv-i \omega \tilde{\gamma}_{n}^{a b c d}\left(\omega ; \mathbf{x}, \mathbf{x}^{\prime}\right)$, that is, $H_{\mathrm{A}_{n}}^{a b c d}\left(x, x^{\prime}\right)=\partial \gamma_{n}^{a b c d}\left(x, x^{\prime}\right) / \partial t$, Eq. (4.7) yields

$$
\widetilde{N}_{n}^{a b c d}\left(\omega ; \mathbf{x}, \mathbf{x}^{\prime}\right)=\omega \operatorname{coth}\left(\frac{\beta \omega}{2}\right) \tilde{\gamma}_{n}^{a b c d}\left(\omega ; \mathbf{x}, \mathbf{x}^{\prime}\right),
$$

or, equivalently,

$$
N_{n}^{a b c d}\left(t, \mathbf{x} ; t^{\prime}, \mathbf{x}^{\prime}\right)=\int_{-\infty}^{\infty} d t^{\prime \prime} J_{\mathrm{FD}}\left(t-t^{\prime \prime}\right) \gamma_{n}^{a b c d}\left(t^{\prime \prime}, \mathbf{x} ; t^{\prime}, \mathbf{x}^{\prime}\right),
$$

where

$$
J_{\mathrm{FD}}(t) \equiv \int_{0}^{\infty} \frac{d \omega}{\pi} \cos (\omega t) \omega \operatorname{coth}\left(\frac{\beta \omega}{2}\right) .
$$

This integral gives a distribution which is singular at $t=0$ and for $t \neq 0$ reduces to $J_{\mathrm{FD}}(t)=-\pi\left[k_{B} T \operatorname{cosech}\left(\pi k_{B} T t\right)\right]^{2}$.

The relations (4.7) or (4.8) [or the equivalent forms (4.11) or (4.12)] have the same form as the fluctuation-dissipation relations which appear in quite general models of quantum mechanics [38-43]. The derivation of these relations is usually done in the framework of linear response theory, in which one considers the response of a quantum system, which is initially at thermal equilibrium, when an external classical time-dependent linear perturbation is "switched on." When evaluating the change in the expectation value of the relevant operator (the operator which couples to the perturbation) induced by the presence of the perturbation, a dissipative term can be identified as the term which changes the sign under a time reversal transformation in the perturbation. This term is characterized by a kernel called the dissipation kernel. It can be shown that the dissipation kernel is related to the fluctuations in equilibrium (in the absence of the perturbation) of the relevant operator by a relation which is exactly the same as Eq. (4.8) or Eq. (4.7). This is the fluctuation-dissipation relation. Using this linear response theory approach, the same fluctuation-dissipation relation has also been derived for some models of quantum many-body systems $[44,43]$ or quantum fields $[42,45]$ coupled to external classical fields. 
This fluctuation-dissipation relation appears also in the context of quantum Brownian motion (or "semiclassical" Brownian motion), in which one is interested in the dynamics of a macroscopic particle in interaction with a heat bath environment, usually modelized by an infinite set of quantum harmonic oscillators. In these models, when the variable representing the center of mass position of the macroscopic particle decoheres, it can be effectively described as a classical stochastic variable. The equation of motion for this stochastic variable is a linear Langevin equation with a Gaussian stochastic source. The classical variable introduced in linear response theory can be envisaged as the position of the Brownian particle, but now this variable becomes a dynamical stochastic variable. The dissipative term in this Langevin equation is responsible for the irreversible dynamics of the Brownian particle. This term contains a dissipation kernel which is related to the correlator of the stochastic source by the relations (4.8) or (4.7) $[15,16]$. This is again the fluctuation-dissipation relation. There are also some models in which a purely quantum description of the Brownian particle is considered $[63,64]$. The dynamics of this particle is then described by a quantum operator in the Heisenberg picture. By elimination of all the environment degrees of freedom in the equation of motion for this operator, one finds a quantum Langevin equation with quantum fluctuating and dissipative terms. These terms are again related by a fluctuation-dissipation relation of the form (4.8) or (4.7).

These analogies allow us to identify the equivalent relations (4.8) and (4.7), and the analogous relations for the physical kernels (4.10), as the fluctuation-dissipation relation in our context. Because of this relation, the kernel $H_{\mathrm{A}}^{a b c d}\left(x, x^{\prime}\right)$ shall be called the dissipation kernel. The same fluctuation-dissipation relation was derived by Mottola [47] in the context of quantum field theory in curved spacetime using the linear response theory approach. This author considered the case in which the background spacetime is static, but his result is easily generalized to a stationary background. In this paper, we have derived the same relation in the context of a Langevin equation for stochastic metric perturbations, which would presumably describe the effective dynamics of gravitational fluctuations after a process of decoherence. For the particular case of a massless scalar field in a Minkowski background, this fluctuation-dissipation relation was derived in Refs. [23,24] from an explicit evaluation of the kernels.

It is clear that the kernel $N^{a b c d}\left(x, x^{\prime}\right)$ describes fluctuations in exactly the same sense as the quantum-mechanical models described above. In fact, as it was pointed out by Mottola [47] from the point of view of linear response theory, it gives the fluctuations in equilibrium of the stressenergy operator. Alternatively, as we have shown in the previous sections, it gives the two-point correlation function of the Gaussian stochastic source in the semiclassical EinsteinLangevin equation. However, the term containing the "dissipation" kernel $H_{\mathrm{A}}^{a b c d}\left(x, x^{\prime}\right)$ in the Einstein-Langevin equation does not generally change sign under a time-reversal transformation in the metric perturbations.

\section{Zero temperature limit}

A state of the scalar field which is of special interest is that described by $\hat{\rho}^{\mathrm{S}}\left(t_{i}\right)=\hat{\rho}[g]=|0\rangle\langle 0|$, where $|0\rangle$ is the vacuum state. This vacuum state can be obtained as the zero temperature limit, $T \rightarrow 0$, of the previous thermal state. The fluctuation-dissipation relation for this state is easily obtained by setting $T=0$ in expression (4.7) or (4.8). We find $\widetilde{H}_{\mathrm{A}_{n}}^{a b c d}\left(\omega ; \mathbf{x}, \mathbf{x}^{\prime}\right)=-i \operatorname{sgn} \omega \widetilde{N}_{n}^{a b c d}\left(\omega ; \mathbf{x}, \mathbf{x}^{\prime}\right)$, or, equivalently, it has the form (4.8), with

$$
K_{\mathrm{FD}}(t)=-i \int_{-\infty}^{\infty} \frac{d \omega}{2 \pi} e^{-i \omega t} \operatorname{sgn} \omega=-\frac{1}{\pi} \mathrm{P}\left(\frac{1}{t}\right) .
$$

This fluctuation-dissipation relation in the alternative form (4.11) reads $\widetilde{N}_{n}^{a b c d}\left(\omega ; \mathbf{x}, \mathbf{x}^{\prime}\right)=\omega \operatorname{sign} \omega \tilde{\gamma}_{n}^{a b c d}\left(\omega ; \mathbf{x}, \mathbf{x}^{\prime}\right)$, or, it has the form (4.12), with

$$
J_{\mathrm{FD}}(t)=\int_{0}^{\infty} \frac{d \omega}{\pi} \cos (\omega t) \omega=-\frac{1}{\pi} \mathcal{P} f\left(\frac{1}{t^{2}}\right),
$$

where $\mathcal{P} f\left(1 / t^{2}\right)$ denotes a Hadamard finite part distribution, which is related to $\mathrm{P}(1 / t)$ by $\mathcal{P} f\left(1 / t^{2}\right)=-(d / d t) \mathrm{P}(1 / t)$ (the definitions of these distributions can be found in Refs. [65]).

\section{High temperature limit}

Let us now consider the high temperature limit. This limit can only be performed when there exists a cutoff frequency $\Omega$, such that $\widetilde{N}_{n}^{a b c d}\left(\omega ; \mathbf{x}, \mathbf{x}^{\prime}\right)$ vanishes for $\omega>\Omega$ [by (4.7), $\widetilde{H}_{\mathrm{A}_{n}}^{a b c d}\left(\omega ; \mathbf{x}, \mathbf{x}^{\prime}\right)$ will also vanish for these values of $\left.\omega\right]$. Such a cutoff frequency is usually related to a characteristic cutoff frequency of the environment degrees of freedom. The high temperature limit corresponds to the limit in which $k_{B} T$ $\gg \hbar \Omega$. In this limit (keeping only the leading order contributions), we expect that thermal fluctuations dominate over quantum fluctuations. To study this limit, it is convenient to restore the dependence in $\hbar$ in the previous results. For this, one has to multiply the constants $\alpha_{B}$ and $\beta_{B}$ by $\hbar$ and the kernel $H_{n}^{a b c d}$ by $1 / \hbar$ in the Einstein-Langevin equation (3.36), and change the combination $\beta \omega$ by $\hbar \beta \omega$ in the previous expressions. In this limit, we can approximate $\tanh (\hbar \beta \omega / 2) \simeq \hbar \beta \omega / 2$, and the fluctuation-dissipation relation reduces to

$$
\frac{1}{\hbar} \widetilde{H}_{\mathrm{A}_{n}}^{a b c d}\left(\omega ; \mathbf{x}, \mathbf{x}^{\prime}\right)=-i \frac{\omega}{2 k_{B} T} \widetilde{N}_{n}^{a b c d}\left(\omega ; \mathbf{x}, \mathbf{x}^{\prime}\right),
$$

or, equivalently, since in this case

$$
\begin{gathered}
K_{\mathrm{FD}}(t) \simeq\left(\hbar / 2 k_{B} T\right)(d / d t) \delta(t), \\
\frac{1}{\hbar} H_{\mathrm{A}_{n}}^{a b c d}\left(t, \mathbf{x} ; t^{\prime}, \mathbf{x}^{\prime}\right)=\frac{1}{2 k_{B} T} \frac{\partial}{\partial t} N_{n}^{a b c d}\left(t, \mathbf{x} ; t^{\prime}, \mathbf{x}^{\prime}\right) .
\end{gathered}
$$

Note that $(1 / \hbar) H_{\mathrm{A}_{n}}^{\text {abcd }}$ is the kernel that appears in the Einstein-Langevin equation (3.36) when one writes the dependence in $\hbar$ explicitly. This relation has the same form as 
the classical Green-Kubo fluctuation-dissipation relation which appears either in a classical theory of linear response [66,39] or in a classical theory of Brownian motion [64,67]. Notice, from Eq. (4.17), that in this high temperature limit we can simply take $\gamma_{n}^{a b c d}\left(x, x^{\prime}\right)=\left(\hbar / 2 k_{B} T\right) N_{n}^{a b c d}\left(x, x^{\prime}\right)$.

\section{B. The fluctuation-dissipation relation for conformal fields in a conformally stationary background}

In the case of a massless conformally coupled scalar field ( $m=0$ and $\xi=1 / 6$ ) and a conformally stationary solution of semiclassical gravity [for instance, a Robertson-Walker (RW) spacetime], the fluctuation-dissipation relation derived in the previous subsection can be generalized when the state of the field in the background solution is the conformal vacuum or a thermal state built on the conformal vacuum. In this case, the action (3.1) for the scalar field is conformally invariant. It is convenient to preserve this conformal invariance when working in dimensional regularization. This can be done by changing, in all the previous expressions which involve dimensional regularization, the parameter $\xi$ by the function $\xi(n) \equiv(n-2) /[4(n-1)]$ and, of course, taking $m$ $=0$. In this way, the dimensional regularized stress-tensor operator (3.6) is traceless. Let $\left(\mathcal{M}, \bar{g}_{a b}\right)$ be a $n$ dimensional conformally stationary spacetime, that is, a spacetime with a global timelike conformal Killing vector field $\zeta^{a}: £_{\zeta} \bar{g}_{a b}$ $=(2 / n) \nabla^{c} \zeta_{c} \bar{g}_{a b}$, where $\nabla_{a}$ is the covariant derivative associated to $\bar{g}_{a b}$. This means that the metric $\bar{g}_{a b}$ is conformally related to a stationary metric $g_{a b}: \bar{g}_{a b}(x)=e^{2 \pi(x)} g_{a b}(x)$, where $\varpi(x)$ is a scalar function. As previously, writing $\zeta^{a}$ $=(\partial / \partial t)^{a}$, the semiclassical spacetime can be foliated by Cauchy hypersurfaces $\Sigma_{t}$ and coordinates $(t, \mathbf{x})$ can be assigned to the spacetime points.

There is a "natural" Fock representation based on a decomposition of the field operator $\hat{\Phi}_{n}[\bar{g}]$ in terms of a complete set of modes $\left\{\bar{u}_{k_{n}}(x)\right\}$, solution of the Klein-Gordon equation with metric $\bar{g}_{a b}$, of the form $\bar{u}_{k_{n}}(x)$ $=e^{-(n-2) \varpi(x) / 2} u_{k_{n}}(x)$, where $\left\{u_{k_{n}}(x)\right\}$ is a complete set of mode solutions of the Klein-Gordon equation in $\left(\mathcal{M}, g_{a b}\right)$ which are of positive frequencies $\omega_{k}$ with respect to $\zeta^{a}$.
Hence, in this sense, we can write the field operator as $\hat{\Phi}_{n}[\bar{g}]=e^{-(n-2) \varpi / 2} \hat{\Phi}_{n}[g]$, where $\hat{\Phi}_{n}[g]$ is the field operator in the stationary spacetime $\left(\mathcal{M}, g_{a b}\right)$. Assuming that no infrared divergencies are present, so that this quantum field theory construction is well defined, the conformal vacuum $|0\rangle$ is defined as the vacuum state of the Fock space corresponding to this representation. If $\hat{a}_{k}^{\dagger}$ and $\hat{a}_{k}$ are the creation and annihilation operators on this Fock space, this state satisfies $\hat{a}_{k}|0\rangle=0$. As shown in Appendix $\mathrm{B}$, in this case we can construct a conserved energy operator which can be identified with the Hamiltonian of a field quantized on $\left(\mathcal{M}, g_{a b}\right): \hat{E}[\bar{g}]=\hat{H}[g]=\sum_{k} \omega_{k} \hat{a}_{k}^{\dagger} \hat{a}_{k}$. This energy operator, however, is not a time-evolution generator for the field operator $\hat{\Phi}_{n}[\bar{g}]$; it generates the time-evolution of the conformally related operator $\hat{\Phi}_{n}[g]$. The many-particle states of the Fock space built on the conformal vacuum are eigenstates of this energy operator.

From this energy operator, a state of thermal equilibrium for a conformal scalar field quantized on $\left(\mathcal{M}, \bar{g}_{a b}\right)$ can be defined using the density operator (4.1). Thermal equilibrium states defined in this way were first proposed by Gibbons and Perry [62]. These authors were inspired in a result by Israel [68] in the framework of relativistic kinetic theory, who found that thermal equilibrium distribution functions can be defined for massless particles in conformally stationary spacetimes. A number of applications have been developed in the literature to study finite-temperature effects of quantum conformal fields in RW universes [69] and in twodimensional spacetimes [70].

Let us begin with a solution of the semiclassical Einstein equation (3.10) consisting of a quantum conformal scalar field in a conformally stationary spacetime $\left(\mathcal{M}, \bar{g}_{a b}\right)$, in the thermal state (4.1). Taking into account that the action (3.3) with $m=0$ and $\xi=\xi(n)$ satisfies $S_{m}\left[\bar{g}, \bar{\Phi}_{n}\right]=S_{m}\left[g, \Phi_{n}\right]$, where $\bar{\Phi}_{n} \equiv e^{-(n-2) \varpi / 2} \Phi_{n}$, it is easy to see that $\hat{T}_{n}^{a b}[\bar{g}]$ $=e^{-(n+2) \varpi} \hat{T}_{n}^{a b}[g]$. Therefore, the kernels evaluated in the thermal state at a temperature $T$ can be related to the corresponding kernels for the stationary background $\left(\mathcal{M}, g_{a b}\right)$. For the noise kernel, we have

$$
N_{n}^{a b c d}[\bar{g}]\left(x, x^{\prime}\right)=e^{-(n+2) \varpi(x)} e^{-(n+2) \varpi\left(x^{\prime}\right)} N_{n}^{a b c d}[g]\left(x, x^{\prime}\right),
$$

and the same relation holds for the kernels $H_{\mathrm{S}_{n}}^{a b c d}$ and $H_{\mathrm{A}_{n}}^{a b c d}$. Since the kernels $N_{n}^{a b c d}[g]$ and $H_{\mathrm{A}_{n}}^{a b c d}[g]$ satisfy the relation (4.8) [or, equivalently, (4.7)], this leads to a fluctuationdissipation relation between the kernels $N_{n}^{a b c d}[\bar{g}]$ and $H_{\mathrm{A}_{n}}^{a b c d}[\bar{g}]$. The same relation holds for the physical kernels obtained by taking the limit $n \rightarrow 4$ as in Eq. (4.10). For the conformal vacuum state, which corresponds to $T=0$, the fluctuation-dissipation relation follows directly from the result of Sec. IV A 1. In the particular case of a spatially flat RW solution of semiclassical gravity, this conformal vacuum fluctuation-dissipation relation was obtained before in Ref. [19] after an explicit calculation of the corresponding kernels. The same relation was derived in Ref. [14] in the 
framework of a "reduced" version of the Einstein-Langevin equation inspired in a Bianchi-I type "mini-superspace", model.

\section{Particle creation}

Let us now return to the case in which $\left(\mathcal{M}, g_{a b}\right)$ is stationary, the scalar field has arbitrary mass $m$ and arbitrary coupling parameter $\xi$, and consider the stochastic perturbation $h_{a b}$. Note that $\left(\mathcal{M}, g_{a b}+h_{a b}\right)$ can be viewed as representing an ensemble of spacetimes distributed according to some probability distribution functional. We are in fact considering a scalar field quantized on each of these spacetimes, described by the operator $\hat{\Phi}[g+h]$, and the family of states of the field, described by $\hat{\rho}[g+h]$.

Let $h_{a b}[\xi]$ be a solution to the semiclassical EinsteinLangevin equation, Eq. (2.11), whose moments vanish for times $t<t_{\mathrm{I}}$ or, at least, they vanish "asymptotically" in the remote past $(t \rightarrow-\infty)$. This means that there is a "remote past epoch" ( $t<t_{\mathrm{I}}$ or $\left.t \rightarrow-\infty\right)$ in which $h_{a b}$ behaves deterministically as a zero tensor. In that case, if we take $\hat{\rho}^{\mathrm{S}}\left(t_{i}\right)$ $=|0\rangle\langle 0|$, where $|0\rangle$ is the vacuum of the natural Fock space for the field quantized on $\left(\mathcal{M}, g_{a b}\right)$, and we consider the limit $t_{i} \rightarrow-\infty$, we have $\hat{\rho}[g+h]=\mid 0$,in $\rangle\langle 0$,in $|$, where $\mid 0$,in $\rangle$ represents the family of "in" vacua for the field quantized on $\left(\mathcal{M}, g_{a b}+h_{a b}\right)$. Treating $h_{a b}$ as a classical "external", perturbation, one could construct a Hamiltonian operator $\hat{H}[g+h](t)$ in the Heisenberg picture for which $\mid 0$,in $\rangle$ would be the ground state in the "remote past epoch.' However, at later times, due to the presence of the perturbation $h_{a b}$, this "in" vacuum state will generally not be the ground state of the Hamiltonian. One then says that "particles" are created in the "in" vacuum.

Physically meaningful many particle "out' states, in particular, an 'out' vacuum $\mid 0$,out $\rangle$ for the scalar field in each of the spacetimes $\left(\mathcal{M}, g_{a b}+h_{a b}\right)$, can be defined if there is also a "far future epoch" for which $h_{a b}$ vanishes (in the same statistical sense as above), either in an exact way for $t>t_{\mathrm{F}}$ or "asymptotically" for $t \rightarrow+\infty$. When this is the case, the vacuum persistence amplitude $\langle 0$,out $| 0$,in $\rangle[g+h]$ $\equiv e^{i W[g+h]}$ is given by the following path integral:

$$
\left.e^{i W[g+h]}=\int \mathcal{D}\left[\Phi_{n}\right]\left\langle 0, \text { out } \mid \Phi_{n}\left(t_{2}\right), t_{2}\right\rangle[g+h]\left\langle\Phi_{n}\left(t_{1}\right), t_{1}\right| 0, \text { in }\right\rangle[g+h] e^{i S_{m}\left[g+h, \Phi_{n}\right]},
$$

where $\left|\varphi_{n}, t_{1}\right\rangle$ and $\left|\varphi_{n}, t_{2}\right\rangle$ denote, respectively, eigenstates of the field operator $\hat{\Phi}_{n}[g+h](t, \mathbf{x})$ at some arbitrary times $t=t_{1}$ and $t=t_{2}$, where $t_{2}>t_{1}$, with eigenvalue $\varphi_{n}(\mathbf{x})$, and where the integration domain for the action is between $t_{1}$ and $t_{2}$. The wave functionals $\left\langle\varphi_{n}, t_{1}\right| 0$,in $\rangle$ and $\left\langle\varphi_{n}, t_{2}\right| 0$,out $\rangle$ have in general a dependence on the metric, which we have indicated in Eq. (4.19). In the limit $t_{1} \rightarrow-\infty$ and $t_{2} \rightarrow+\infty$, these wave functionals do not depend on the perturbation $h_{a b}$. The total probability of particle creation is given by [71]

$$
P[h ; g]=2 \lim _{n \rightarrow 4} \operatorname{Im} W[g+h] .
$$

One can show that $\operatorname{Im} W$ is free of ultraviolet divergencies in the limit $n \rightarrow 4$, and that it is always positive or zero, so that the probability $P$ is well defined by this expression.

As we have done in the previous section for the influence action, we can now expand the action $W[g+h]$ in the perturbation $h_{a b}$. In order to do so, one has to evaluate the functional derivatives of $W[g+h]$ in the background metric $g_{a b}$. Using Eq. (4.19), these derivatives can be related to "in-out" matrix elements of operators in the background. Since $g_{a b}$ is stationary, the "in" and "out" vacua in the background must be identified with the natural vacuum $|0\rangle$. Therefore, these background "in-out" matrix elements become expectation values in the state $|0\rangle$. It is then easy to see that the expansion of $W[g+h]$ in the metric perturbation $h_{a b}$ is equal to that of $S_{\mathrm{IF}}\left[g+h^{+}, g+h^{-}\right]$with $h_{a b}^{+}=h_{a b}$ and $h_{a b}^{-}=0$, and taking the expectation values in $|0\rangle$. In particular, from the imaginary part of this expansion [see Eq. (3.18)], we get

$$
P[h ; g]=\int d^{4} x d^{4} y \sqrt{-g(x)} \sqrt{-g(y)} h_{a b}(x) N^{a b c d}[g](x, y) h_{c d}(y)+0\left(h^{3}\right),
$$

where $N^{a b c d}$ is the zero temperature physical noise kernel defined in Eq. (4.10). This physical noise kernel is related to the lowest order quantum stress-energy fluctuations in vacuum by (2.9). Note that the higher order corrections in
Eq. (4.21) would contain higher order vacuum stress-energy fluctuations. Equation (4.21) is a generalization of an expression derived by Sexl and Urbantke [72] for the total probability of particle creation by metric perturbations around 
Minkowski spacetime.

Equation (4.21) gives also the expectation value of the number operator for "out" particles in the "in' vacuum, computed to lowest order in the metric perturbation. In order to show this, let us expand the scalar field action as the action in the stationary background plus interaction terms (the terms containing the metric perturbation). The interaction term to lowest order in $h_{a b}$ is

$$
S^{(1)}=\int d^{n} x \mathcal{L}_{n}^{(1)}\left[\Phi_{n}, h ; g\right]
$$

with

$$
\mathcal{L}_{n}^{(1)}=(1 / 2) \sqrt{-g} T^{a b}\left[g, \Phi_{n}\right] h_{a b}
$$

In order to construct the $S$-matrix operator, we need the interaction Hamiltonian density operator in the interaction picture. Note that the field and canonical momentum operators in the interaction picture can be identified with the operators $\hat{\Phi}_{n}[g]$ and $\hat{\Pi}_{n}[g]$, respectively. Following Appendix B, we can obtain the canonical Hamiltonian density for the metric $g_{a b}+h_{a b}$ and work out the interaction term to first order in the metric perturbation. Although in this case the interaction Lagrangian density depends on the derivatives of the field, we find that, to first order in $h_{a b}$, the interaction Hamiltonian density operator in the interaction picture is given by $-\mathcal{L}_{n}^{(1)}\left[\hat{\Phi}_{n}[g], h ; g\right]$. Hence, to first order in the metric perturbation, the $S$-matrix operator is given by $\hat{S}=1+\hat{S}^{(1)}$ $+O\left(h^{2}\right)$, where

$$
\hat{S}^{(1)}=\frac{i}{2} \int d^{n} x \sqrt{-g} \hat{T}_{n}^{a b}[g] h_{a b} .
$$

The expectation value of the "out" particle number operator, $\hat{N}^{\text {out }}$, in the "in" vacuum (in the Heisenberg picture) is given by $N[h ; g] \equiv\left\langle 0\right.$,in $\left|\hat{N}^{\text {out }}\right| 0$,in $\rangle=\left\langle 0\left|\hat{S}^{\dagger} \hat{N} \hat{S}\right| 0\right\rangle$, where $\hat{N}$ is the particle number operator in the background $\hat{N}$ $\equiv \Sigma_{k} \hat{a}_{k}^{\dagger} \hat{a}_{k}$. To lowest order, we have

$$
N[h ; g]=\sum_{k, p}\left|\left\langle 1_{k}, 1_{p}\left|\hat{S}^{(1)}\right| 0\right\rangle\right|^{2}+O\left(h^{3}\right),
$$

where $\left|1_{k}, 1_{p}\right\rangle$ is the two-particle state $\left|1_{k}, 1_{p}\right\rangle \equiv \hat{a}_{k}^{\dagger} \hat{a}_{p}^{\dagger}|0\rangle$. Clearly, since $\hat{S}^{(1)}$ is quadratic in the field operator, at this order $N$ can also be written as

$$
\begin{aligned}
N / 2= & \sum_{n}\left\langle 0\left|\hat{S}^{(1) \dagger}\right| n\right\rangle\left\langle n\left|\hat{S}^{(1)}\right| 0\right\rangle \\
& -\left\langle 0\left|\hat{S}^{(1) \dagger}\right| 0\right\rangle\left\langle 0\left|\hat{S}^{(1)}\right| 0\right\rangle+O\left(h^{3}\right)
\end{aligned}
$$

where $\{|n\rangle\}$ represents the complete orthonormal basis of the Fock space. Using Eq. (4.22), this last expression can be written in terms of the vacuum noise kernel $N_{n}^{a b c d}[g](x, y)$ [see Eq. (3.14)]. Taking the limit $n \rightarrow 4$, we see that the expression for one half of the number of created particles
$N[h ; g] / 2$ to lowest order in the metric perturbation coincides with that for $P[h ; g]$ in Eq. (4.21).

The energy of the created particles, defined as $E[h ; g]$ $\equiv\left\langle 0\right.$,in $\left|\Sigma_{k} \omega_{k} \hat{N}_{k}^{\text {out }}\right| 0$,in $\rangle=\left\langle 0\left|\hat{S}^{\dagger} \hat{H} \hat{S}\right| 0\right\rangle$, where $\hat{N}_{k}^{\text {out }}$ is the " "out" number operator in the $k$ mode and $\hat{H}=\Sigma_{k} \omega_{k} \hat{a}_{k}^{\dagger} \hat{a}_{k}$ is the Hamiltonian operator in the background, is similarly given by

$$
E[h ; g]=\frac{1}{2} \sum_{k, p}\left(\omega_{k}+\omega_{p}\right)\left|\left\langle 1_{k}, 1_{p}\left|\hat{S}^{(1)}\right| 0\right\rangle\right|^{2}+O\left(h^{3}\right) \text {. }
$$

Comparison of Eq. (4.24) with Eqs. (4.23) and (4.21), suggests that it may be possible in some cases to write this last expression (in the limit $n \rightarrow 4$ ) in terms of the Fourier transform of the vacuum noise kernel.

As an example, let us consider the case when $\left(\mathcal{M}, g_{a b}\right)$ is simply Minkowski spacetime, $\left(\mathbb{R}^{4}, \eta_{a b}\right)[31,73]$, which is the trivial solution of semiclassical gravity. Working in a global inertial coordinate system $\left\{x^{\mu}\right\}$, in this case the kernels depend only on the difference $(x-y)^{\mu}$ and, thus, we can define their Fourier transforms as $K(x-y)$ $\equiv(2 \pi)^{-4} \int d^{4} p e^{i p \cdot(x-y)} \widetilde{K}(p)$, where $p \cdot x \equiv \eta_{\mu \nu} p^{\mu} x^{\nu}$. Introducing the Fourier transform of $h_{a b}(x)$ in a similar way [note that $\left.\tilde{h}_{a b}(-p)=\widetilde{h}_{a b}^{*}(p)\right]$, Eq. (4.21) can be written as

$$
P[h ; \eta]=\int \frac{d^{4} p}{(2 \pi)^{4}} \tilde{N}^{a b c d}(p) \tilde{h}_{a b}^{*}(p) \tilde{h}_{c d}(p)+O\left(h^{3}\right) .
$$

On the other hand, the energy of the created particles is given by $[74]$

$$
\begin{aligned}
E[h ; \eta]= & 2 \int \frac{d^{4} p}{(2 \pi)^{4}} p^{0} \theta\left(p^{0}\right) \tilde{N}^{a b c d}(p) \widetilde{h}_{a b}^{*}(p) \tilde{h}_{c d}(p) \\
& +O\left(h^{3}\right) .
\end{aligned}
$$

The vacuum noise and dissipation kernels for a Minkowski background can be written in terms of two pairs of scalar kernels, $N_{r}(x-y)$ and $D_{r}(x-y)$, respectively, with $r=1,2$ [73] (see also Ref. [31] for a particular case in which $N_{2}$ $\left.=D_{2}=0\right)$. Each pair of kernels $\left(N_{r}, D_{r}\right)$ satisfies the fluctuation-dissipation relation found in Sec. IV A 1. One finds $[71,74]$ that

$$
\begin{aligned}
\widetilde{N}^{a b c d}(p) \widetilde{h}_{a b}^{*}(p) \widetilde{h}_{c d}(p)= & \widetilde{C}_{a b c d}^{(1)}(p) \widetilde{C}^{(1) * a b c d}(p) \widetilde{N}_{1}(p) \\
& +\left|\widetilde{R}^{(1)}(p)\right|^{2} \widetilde{N}_{2}(p)
\end{aligned}
$$

where $\widetilde{C}_{a b c d}^{(1)}(p), \widetilde{R}^{(1)}(p)$ and $\tilde{N}_{r}(p)$ are, respectively, the Fourier transforms of the linearized Weyl tensor, the scalar curvature and the kernels $N_{r}(x-y), \quad r=1,2 ; \tilde{N}_{r}(p)$ depend only on $p^{2} \equiv \eta_{\mu \nu} p^{\mu} p^{\nu}$. It is then easy to see, using the fluctuation-dissipation relation, that 


$$
E[h ; \eta]=i \int \frac{d^{4} p}{(2 \pi)^{4}} p^{0}\left[\widetilde{C}_{a b c d}^{(1)}(p) \widetilde{C}^{(1) * a b c d}(p) \widetilde{D}_{1}(p)+\left|\widetilde{R}^{(1)}(p)\right|^{2} \widetilde{D}_{2}(p)\right]+O\left(h^{3}\right) .
$$

Hence, in the case of a Minkowski background, the energy of the created particles can be expressed in terms of the dissipation kernels $D_{1}$ and $D_{2}$ for the Minkowskian vacuum. It is not clear, however, that, for other stationary backgrounds, the energy of the created particles can be related to dissipation in vacuum in a similar way.

The probability of particle creation (4.21) is a fluctuating quantity, due to the functional dependence on the stochastic perturbation $h_{a b}$. We may compute its averaged value $\langle P[h ; g]\rangle_{c}$, which [neglecting the higher order corrections in Eq. (4.21)] is given by

$$
\langle P[h ; g]\rangle_{c}=P\left[\langle h\rangle_{c} ; g\right]+\int d^{4} x d^{4} y \sqrt{-g(x)} \sqrt{-g(y)} N^{a b c d}[g](x, y)\left\langle h_{a b}^{\mathrm{f}}(x) h_{c d}^{\mathrm{f}}(y)\right\rangle_{c},
$$

where $h_{a b}^{\mathrm{f}} \equiv h_{a b}-\left\langle h_{a b}\right\rangle_{c}$. The first term in the right hand side of Eq. (4.29) is the probability of particle creation (or one half of the number of created particles) that one would obtain in the spacetime $\left(\mathcal{M}, g_{a b}+\left\langle h_{a b}\right\rangle_{c}\right)$. The second term will be greater than zero when stress-energy fluctuations are present $^{3}$ since, from the Einstein-Langevin equation, this implies $\left\langle h_{a b}^{\mathrm{f}}(x) h_{c d}^{\mathrm{f}}(y)\right\rangle_{c} \neq 0$. Note that, when this is the case, from the fluctuation-dissipation relation of Sec. IV A 1, the vacuum dissipation kernel will be also non-vanishing. Hence, metric fluctuations induced by matter stress-energy fluctuations generally increase the mean value of the number of created particles with respect to the same quantity in the "perturbed" semiclassical spacetime $\left(\mathcal{M}, g_{a b}+\left\langle h_{a b}\right\rangle_{c}\right)$.

The above result for the total probability of particle creation and number of created particles can be easily generalized to the case of a massless conformally coupled scalar field and a conformally stationary semiclassical background. When this background is a spatially flat RW universe [19], performing conformal transformations in the metric perturbations and in the kernels as in Eq. (4.18), one gets expressions analogous to Eqs. (4.25), (4.27) and (4.28) with $\mathrm{N}_{2}$ $=D_{2}=0$ (see Refs. [56,19,31] for more details).

\section{CONCLUSIONS}

In the first part of this paper, we have shown how a consistent stochastic semiclassical theory of gravity can be formulated. This theory is a perturbative generalization of semiclassical gravity which describes the back reaction of the lowest order stress-energy fluctuations of quantum matter fields on the gravitational field through the semiclassical Einstein-Langevin equation. We have shown that this equation can be formally derived with a method based on the influence functional of Feynman and Vernon, where one considers the metric field as the "system" of interest and the matter fields as part of its "environment" [9]. Our approach clarifies the physical meaning of the semiclassical Langevintype equations previously derived with the same functional

\footnotetext{
${ }^{3}$ Except in some rare cases, for which $N^{a b c d}(x, y)$ is not strictly positive definite and $\left\langle h_{a b}^{\mathrm{f}}(x) h_{c d}^{\mathrm{f}}(y)\right\rangle_{c}$ is such that it "hits" the zero eigenvalue.
}

method [12-14,19-25], since it links the source of stochastic fluctuations to quantum matter stress-energy fluctuations, and allows us to formulate the theory in a general way. At the same time, we have also developed a method to compute the semiclassical Einstein-Langevin equation using dimensional regularization. This provides an alternative and more direct way of computing the equation with respect to the previous calculations, based on a specific evaluation of the effective action of Feynman and Vernon [12-14,19-25]. In a subsequent paper [73], we shall apply this method to solve the Einstein-Langevin equation around some simple solutions of semiclassical gravity.

The second part of the paper was devoted to the existence of fluctuation-dissipation relations and to particle creation in the context of stochastic semiclassical gravity. When the background solution of semiclassical gravity consists of a stationary spacetime and a scalar field in a thermal equilibrium state, we have identified a dissipation kernel in the Einstein-Langevin equation which is related to the noise kernel by a fluctuation-dissipation relation. The same relation was previously derived by Mottola [47] using a linear response theory approach. We have also generalized this result to the case of a conformal scalar field in a conformally stationary background solution of semiclassical gravity.

Our analysis seems to indicate that for a fluctuationdissipation relation to be present in stochastic semiclassical gravity, the semiclassical background solution must satisfy certain conditions. In this paper we have just analyzed the simplest cases for which such a relation exists. Further work must be done to investigate whether a similar relation is present in other situations of physical interest, such as black hole backgrounds $[48,49,24]$, or non-conformal fields in RW backgrounds in the instantaneous vacua or the thermal states defined in Ref. [75].

We have also studied particle creation by stochastic metric perturbations in stationary and conformally stationary (for conformal matter fields in this latter case) background solutions of semiclassical gravity. We have expressed the total probability of particle creation and the number of created particles (the expectation value of the number operator for "out" particles in the "in" vacuum) in terms of the vacuum noise kernel. We have shown that the averaged value of those quantities is enhanced by the presence of stochastic 
metric fluctuations. In the particular cases of a Minkowski background and a conformal field in a spatially flat RW background, the energy of the created particles can be expressed in terms of the vacuum dissipation kernels.

It should be stressed that the concept of particle creation is only well defined when the solutions of the EinsteinLangevin equation vanish in the "remote past" and in the "far future" (at least, "asymptotically"). However, there can be physically meaningful solutions of the EinsteinLangevin equation that do not satisfy these rather strong conditions. In this case, vacuum noise and dissipation in stochastic semiclassical gravity can include effects that are not associated to particle creation.

\section{ACKNOWLEDGMENTS}

We are grateful to Esteban Calzetta, Jaume Garriga, BeiLok Hu, Ted Jacobson and Albert Roura for very helpful suggestions and discussions. This work has been partially supported by the CICYT Research Project number AEN980431, and the European Project number CI1-CT94-0004.

\section{APPENDIX A: EXPANSION OF THE STRESS-ENERGY TENSOR AROUND A BACKGROUND METRIC}

The expansion of the stress-energy tensor functional

$$
\begin{aligned}
T^{a b}\left[g, \Phi_{n}\right] \equiv & \nabla^{a} \Phi_{n} \nabla^{b} \Phi_{n}-\frac{1}{2} g^{a b} \nabla^{c} \Phi_{n} \nabla_{c} \Phi_{n} \\
& -\frac{1}{2} g^{a b} m^{2} \Phi_{n}^{2}+\xi\left(g^{a b} \square-\nabla^{a} \nabla^{b}+G^{a b}\right) \Phi_{n}^{2},
\end{aligned}
$$

around a background metric $g_{a b}$ is given by

$$
T^{a b}\left[g+h, \Phi_{n}\right]=T^{a b}\left[g, \Phi_{n}\right]+T^{(1) a b}\left[g, \Phi_{n} ; h\right]+0\left(h^{2}\right),
$$

with

$$
\begin{aligned}
T^{(1) a b}\left[g, \Phi_{n} ; h\right]= & -T^{a c}\left[g, \Phi_{n}\right] h_{c}^{b}-T^{b c}\left[g, \Phi_{n}\right] h_{c}^{a}-\frac{1}{2}\left(\nabla^{c} \Phi_{n} \nabla_{c} \Phi_{n}+m^{2} \Phi_{n}^{2}\right) h^{a b}+\frac{1}{2} g^{a b} \nabla^{c} \Phi_{n} \nabla^{d} \Phi_{n} h_{c d} \\
& +\frac{\xi}{2}\left[-R h^{a b}+g^{a b} R^{c d} h_{c d}+\nabla^{c} \nabla^{a} h_{c}^{b}+\nabla^{c} \nabla^{b} h_{c}^{a}-\nabla^{a} \nabla^{b} h_{c}^{c}-\square h^{a b}+g^{a b}\left(\square h_{c}^{c}-\nabla^{c} \nabla^{d} h_{c d}\right)\right. \\
& \left.+\left(\nabla^{a} h_{c}^{b}+\nabla^{b} h_{c}^{a}-\nabla_{c} h^{a b}-2 g^{a b} \nabla^{d} h_{c d}+g^{a b} \nabla_{c} h_{d}^{d}\right) \nabla^{c}+2 h^{a b} \square-2 g^{a b} h_{c d} \nabla^{c} \nabla^{d}\right] \Phi_{n}^{2},
\end{aligned}
$$

where the covariant derivatives and curvature tensors are those of the metric $g_{a b}$, and indices are raised with inverse background metric $g^{a b}$.

\section{APPENDIX B: HAMILTONIAN OPERATOR IN A STATIONARY SPACETIME AND THERMAL WICK'S THEOREM}

In this appendix, we construct the Hamiltonian or energy operator for a quantum scalar field in a stationary spacetime. For a more rigorous mathematical treatment, see Ref. [60] and, for the particular case of a static spacetime, see Ref. [3]. We also show how this construction can be generalized for a conformal scalar field in a conformally stationary spacetime. Using this Hamiltonian to define a thermal density operator, we shall see how thermal four-point functions can be expressed in terms of thermal two-point functions ("thermal Wick's theorem"').

Let $\left(\mathcal{M}, g_{a b}\right)$ be a $n$ dimensional stationary spacetime, that is, a spacetime with a global timelike Killing vector field $\zeta^{a} \equiv(\partial / \partial t)^{a}$, and consider a linear real scalar field $\Phi_{n}$ on it. Assuming that the spacetime is globally hyperbolic, we can foliate it by a family of Cauchy hypersurfaces $\Sigma_{t}$, labeled by the Killing time $t$ (hypersurfaces of constant $t$ ), and give coordinates to each point of the spacetime $x^{\mu}=(t, \mathbf{x})$, where $\mathbf{x} \equiv\left(x^{i}\right)$ are local coordinates on each of these hypersurfaces. Let $n^{a}$ be the future directed unit (i.e., $n_{a} n^{a}=-1$ and $n^{t}$
$>0$ ) vector field normal to each hypersurface $\Sigma_{t}$. The induced metric on each $\Sigma_{t}$ by the spacetime metric is $q_{a b}$ $\equiv g_{a b}+n_{a} n_{b}$ [50]; then $q_{b}^{a}$ is a projector orthogonal to $n^{a}$. We can decompose the Killing vector into its normal and tangential parts to each $\Sigma_{t}$ : $\zeta^{a}=N n^{a}+N^{a}$, where $N \equiv$ $-\zeta^{a} n_{a}$ and $N^{a} \equiv q_{b}^{a} \zeta^{b}$ are, respectively, the lapse function and the shift vector. In the basis associated to the coordinate system $\left\{x^{\mu}\right\}$, the components $g_{\mu \nu}$ of the metric are independent of $t$ and can be written as $g_{t t}=-N^{2}+N_{i} N^{i}, g_{t i}=N_{i}$, $g_{i j}=q_{i j}$, with $N_{i}=q_{i j} N^{j}$. One can also write $\sqrt{-g}=N \sqrt{q}$, where $q \equiv \operatorname{det}\left(q_{i j}\right)$.

To construct the classical Hamiltonian, we write the Lagrangian density as

$$
\mathcal{L}_{n}=\frac{1}{2} \sqrt{q} N\left[\left(n^{\mu} \partial_{\mu} \Phi_{n}\right)^{2}-q^{i j} \partial_{i} \Phi_{n} \partial_{j} \Phi_{n}-\left(m^{2}+\xi R\right) \Phi_{n}^{2}\right],
$$

where $n^{t}=1 / N, n^{i}=-N^{i} / N$ and $q^{i j}$ is the inverse of $q_{i j}$, $q_{i k} q^{k j}=\delta_{i}^{j}$. The momentum canonical conjugate to $\Phi_{n}$ is $\Pi_{n}=\sqrt{q} n^{\mu} \partial_{\mu} \Phi_{n}$ and the Hamiltonian density is constructed as usual, $\mathcal{H}_{n}=\Pi_{n} \partial_{t} \Phi_{n}-\mathcal{L}_{n}$, from which the Hamiltonian functional on the hypersurface $\Sigma_{t}$ is given by $H(t)$ $=\int_{\Sigma_{t}} d^{n-1} \mathbf{x} \mathcal{H}_{n}(x)$. Integrating by parts and dropping surface terms, we get 


$$
H(t)=\frac{1}{2} \int_{\Sigma_{t}} d^{n-1} \mathbf{x} \sqrt{q}\left\{-\Phi_{n}\left[\left(D^{i} N\right) D_{i}+N\left(\Delta-m^{2}-\xi R\right)\right] \Phi_{n}-\Phi_{n}\left(D_{i} N^{i}+N^{i} D_{i}\right)\left(\frac{\Pi_{n}}{\sqrt{q}}\right)+\frac{\Pi_{n}}{\sqrt{q}} N^{i} D_{i} \Phi_{n}+N\left(\frac{\Pi_{n}}{\sqrt{q}}\right)^{2}\right\},
$$

where $D_{i}$ is the covariant derivative on the $n-1$ dimensional Riemannian spaces $\left(\Sigma_{t}, q_{i j}\right)$ (associated to the metric $q_{i j}$ ), and $\Delta \equiv D^{i} D_{i}$ is the associated Laplace-Beltrami operator. For a field $\Phi_{n}$ and its momentum conjugate $\Pi_{n}$ satisfying the Hamiltonian equations of motion, this Hamiltonian is a conserved quantity, i.e., it is independent of $t$.

The analogous quantity in the Lagrangian formalism is the canonical energy functional, which is defined in terms of the canonical stress-energy tensor functional,

$$
\begin{aligned}
T_{a b}^{\mathrm{can}}\left[g, \Phi_{n}\right] \equiv & \nabla_{a} \Phi_{n} \nabla_{b} \Phi_{n}-\frac{1}{2} g_{a b} \nabla^{c} \Phi_{n} \nabla_{c} \Phi_{n} \\
& -\frac{1}{2} g_{a b}\left(m^{2}+\xi R\right) \Phi_{n}^{2}
\end{aligned}
$$

as $E_{\text {can }} \equiv \int_{\Sigma} d \Sigma n^{a} \zeta^{b} T_{a b}^{\text {can }}\left[g, \Phi_{n}\right]$, where $\Sigma$ is a Cauchy hypersurface, $n^{a}$ is the future directed unit vector field normal to $\Sigma$, and $d \Sigma$ is the invariant volume element on $\Sigma$ constructed with the metric induced by $g_{a b}$. By Noether's theorem [50], this energy functional is conserved, i.e., it is independent of the choice of Cauchy hypersurface $\Sigma$, when $\Phi_{n}$ satisfies the Klein-Gordon equation. Choosing $\Sigma_{t}$ as the Cauchy hypersurface, we can obtain an expression for $E_{\text {can }}$ after the substitution of $\Pi_{n}$ by $\sqrt{q} n^{\mu} \partial_{\mu} \Phi_{n}$ in the Hamiltonian (B2). Note that we can also introduce an energy functional $E$ $\equiv \int_{\Sigma} d \Sigma n^{a} \zeta^{b} T_{a b}\left[g, \Phi_{n}\right]$, where $T_{a b}$ is the stress-energy tensor functional (2.2) [50]. For a field $\Phi_{n}$ satisfying the KleinGordon equation, this is also a conserved quantity. However, choosing a Cauchy hypersurface $\Sigma_{t}$, one can show that $n^{a} \zeta^{b}\left(T_{a b}-T_{a b}^{\mathrm{can}}\right)$ is a divergence on the space $\left(\Sigma_{t}, q_{i j}\right)$ and, thus, dropping surface terms, we have $E=E_{\text {can }}$.

We can now formally construct the Hamiltonian "operator" in the Heisenberg picture simply by replacing $\Phi_{n}$ and $\Pi_{n}$ by their corresponding operators $\hat{\Phi}_{n}$ and $\hat{\Pi}_{n}$ in Eq. (B2) and using, as always, a Weyl ordering prescription for the operators. This operator is a conserved quantity, that is, it is independent of the time $t$; therefore, it is equal to the Hamiltonian operator in the Schrödinger picture and we simply denote it by $\hat{H}$. Since the momentum operator in the Heisenberg picture satisfies $\hat{\Pi}_{n}=\sqrt{q} n^{\mu} \partial_{\mu} \hat{\Phi}_{n}$, this Hamiltonian operator can also be obtained from the canonical energy functional [hence, $\hat{H}$ represents also a conserved energy operator]. Taking into account that the field operator $\hat{\Phi}_{n}$ satisfies the Klein-Gordon equation, we find

$$
\begin{aligned}
\hat{H}= & \frac{1}{4} \int_{\Sigma_{t}} d^{n-1} \mathbf{x} \sqrt{q} \frac{1}{N}\left[\left\{\partial_{t} \hat{\Phi}_{n},\left(\partial_{t} \hat{\Phi}_{n}-N^{i} \partial_{i} \hat{\Phi}_{n}\right)\right\}\right. \\
& \left.-\left\{\hat{\Phi}_{n}, \partial_{t}\left(\partial_{t} \hat{\Phi}_{n}-N^{i} \partial_{i} \hat{\Phi}_{n}\right)\right\}\right] .
\end{aligned}
$$

In this case, there exists a natural Fock representation based on a decomposition of the field operator $\hat{\Phi}_{n}$ in terms of a complete set of modes $\left\{u_{k_{n}}(x)\right\}$, solution of the KleinGordon equation, which have positive frequency with respect to the Killing vector $\zeta^{a} \equiv(\partial / \partial t)^{a}: \partial_{t} u_{k_{n}}(x)$ $=-i \omega_{k} u_{k_{n}}(x)$, with $\omega_{k}>0$. The label $k$ of each mode must in general be understood as representing a set of discrete or continuous indices, and, thus, the summations over $k$ represent either a discrete sum or an integral with some suitable measure (or a combination of these two possibilities). We assume that these modes have the same physical dimensions as the field $\Phi_{n}$ (this is the reason why we put a subindex $n$ ). These modes have to be orthonormal with respect to the inner product $\left(\phi_{1}, \phi_{2}\right) \equiv-i \int_{\Sigma} d \Sigma n^{a}\left(\phi_{1} \partial_{a} \phi_{2}^{*}-\phi_{2}^{*} \partial_{a} \phi_{1}\right)$, which is independent of the Cauchy hypersurface $\Sigma$ when $\phi_{1}$ and $\phi_{2}$ are solutions of the Klein-Gordon equation $[4,76]$, i.e., $\left(u_{k_{n}}, u_{l_{n}}\right)=\delta_{k l}$ and $\left(u_{k_{n}}, u_{l_{n}}^{*}\right)=0$.

The field operator can then be written as

$$
\hat{\Phi}_{n}(x)=\sum_{k}\left[u_{k_{n}}(x) \hat{a}_{k}+u_{k_{n}}^{*}(x) \hat{a}_{k}^{\dagger}\right]
$$

where $\hat{a}_{k}^{\dagger}$ and $\hat{a}_{k}$ are creation and annihilation operators on the Fock space associated to this mode decomposition, which satisfy the usual commutation relations $[4,76]$. Using these commutation relations and the orthonormality conditions for the modes evaluated on $\Sigma_{t}$, substituting Eq. (B5) into Eq. (B4), one finds the Fock space representation of the formal Hamiltonian "operator" $\hat{H}=\Sigma_{k} \omega_{k}\left(\hat{a}_{k}^{\dagger} \hat{a}_{k}+\frac{1}{2}\right)$. We can make this last expression well defined by subtraction of the "divergent" constant c-number $\Sigma_{k}\left(\omega_{k} / 2\right)$, that is, we can introduce a renormalized Hamiltonian operator as

$$
\hat{H}_{R}=\sum_{k} \omega_{k} \hat{a}_{k}^{\dagger} \hat{a}_{k} .
$$

Note that $\hat{H}_{R}$ is given by an expression similar to Eq. (B4), but adding a normal ordering prescription for the operators $\hat{a}_{k}$ and $\hat{a}_{k}^{\dagger}$, or, equivalently (dropping surface terms), by $\hat{H}_{R}=\int_{\Sigma} d \Sigma n^{a} \zeta^{b}: \hat{T}_{n_{a b}}[g]:=\int_{\Sigma} d \Sigma n^{a} \xi^{b}: \hat{T}_{n_{a b}}^{\mathrm{can}}[g]$ :, where $\hat{T}_{n_{a b}}[g]$ is defined in Eq. (3.6), $\hat{T}_{n_{a b}}^{\mathrm{can}}[g]$ is analogously defined after Eq. (B3), and : : means normal ordering [76,5]. The vacuum and the many-particle states of the Fock space are eigenstates of this Hamiltonian operator with zero and positive eigenvalues, respectively (given by the sum of the $\omega_{k}$ 's corresponding to the particle contents of the state).

From Eq. (B5) and $\hat{\Pi}_{n}=\sqrt{q} n^{\mu} \partial_{\mu} \hat{\Phi}_{n}$, using the positive frequency condition and 


$$
\left[\hat{H}_{R}, \hat{a}_{k}\right]=-\omega_{k} \hat{a}_{k}, \quad\left[\hat{H}_{R}, \hat{a}_{k}^{\dagger}\right]=\omega_{k} \hat{a}_{k}^{\dagger},
$$

we get $\partial_{t} \hat{\Phi}_{n}=i\left[\hat{H}_{R}, \hat{\Phi}_{n}\right]$ and $\partial_{t} \hat{\Pi}_{n}=i\left[\hat{H}_{R}, \hat{\Pi}_{n}\right]$. These are the Heisenberg equations of motion, which are equivalent to the Klein-Gordon equation for the operator $\hat{\Phi}_{n}$. From these equations, we see that the operator $\exp \left(-i \hat{H}_{R}\left(t-t^{\prime}\right)\right)$ generates the time evolution of operators in the Heisenberg picture.

\section{Conformal field in a conformally stationary spacetime}

Let us now consider a massless conformally coupled real scalar field $\bar{\Phi}_{n}$ in a $n$ dimensional spacetime $\left(\mathcal{M}, \bar{g}_{a b}\right)$, assumed to be conformally stationary and globally hyperbolic. The action $S_{m}\left[\bar{g}, \bar{\Phi}_{n}\right]$ for the field is given by Eq. (3.3) with $m=0$ and $\xi=\xi(n) \equiv(n-2) /[4(n-1)]$. In this case, the spacetime has a global timelike conformal Killing vector field $\quad \zeta^{a} \equiv(\partial / \partial t)^{a}$, which satisfies $\quad \bar{\nabla}_{a} \zeta_{b}+\bar{\nabla}_{b} \zeta_{a}$ $=(2 / n) \bar{\nabla}^{c} \zeta_{c} \bar{g}_{a b}$, where $\bar{\nabla}_{a}$ is the covariant derivative associated to the metric $\bar{g}_{a b}$. The metric $\bar{g}_{a b}$ is conformally related to a stationary metric $g_{a b}: \bar{g}_{a b}(x)=e^{2 \pi(x)} g_{a b}(x)$. The foliation of the spacetime by Cauchy hypersurfaces $\Sigma_{t}$ and the coordinates $x^{\mu}=(t, \mathbf{x})$ are introduced as above.

Given a Cauchy hypersurface $\Sigma$, with unit normal $\bar{n}^{a}$ (as above, we take $\bar{n}^{t}>0$ ), we can introduce the energy functional as $E \equiv \int_{\Sigma} \overline{d \Sigma} \bar{n}^{a} \zeta^{b} T_{a b}\left[\bar{g}, \bar{\Phi}_{n}\right]$, where $\overline{d \Sigma}$ is the invariant volume element constructed with the metric on $\Sigma$ induced by the metric $\bar{g}_{a b}$. Given that the stress-energy tensor $T_{a b}\left[\bar{g}, \bar{\Phi}_{n}\right]$ is traceless when the field $\bar{\Phi}_{n}$ satisfies the KleinGordon equation, it is easy to see from the equation for the conformal Killing vector $\zeta^{a}$ that this energy functional is conserved. In fact, choosing a hypersurface $\Sigma_{t}$ to evaluate this energy, and introducing $\Phi_{n} \equiv e^{(n-2) \varpi / 2} \bar{\Phi}_{n}$, it is easy to see [77] that

$$
E=\int_{\Sigma_{t}} d^{n-1} \mathbf{x} \sqrt{q} n^{a} \zeta^{b} T_{a b}\left[g, \Phi_{n}\right],
$$

where $n^{a}$ and $q_{i j}$ are constructed with the metric $g_{a b}$. Thus, $E$ is equal to the energy functional for the field $\Phi_{n}$ in the stationary spacetime $\left(\mathcal{M}, g_{a b}\right)$.

Using the "natural" Fock representation, based on the decomposition of the field operator $\hat{\Phi}_{n}[\bar{g}]$ in terms of modes $\bar{u}_{k_{n}}(x)=e^{-(n-2) \varpi(x) / 2} u_{k_{n}}(x)$, we can construct the renormalized energy operator in the Heisenberg picture, $\hat{E}_{R}[\bar{g}]$, associated to the above energy functional $E$. Here, as above, $\left\{u_{k_{n}}(x)\right\}$ is a complete set of modes, solution of the KleinGordon equation in the stationary spacetime $\left(\mathcal{M}, g_{a b}\right)$, which are of positive frequency with respect to $\zeta^{a} \equiv(\partial / \partial t)^{a}$.
Dropping surface terms, we get $\hat{E}_{R}[\bar{g}]=\Sigma_{k} \omega_{k} \hat{a}_{k}^{\dagger} \hat{a}_{k}$, where $\hat{a}_{k}^{\dagger}$ and $\hat{a}_{k}$ are creation and annihilation operators on the Fock space associated to these conformal modes.

Alternatively, one can perform the transformation $\bar{\Phi}_{n}$ $\equiv e^{-(n-2) \varpi / 2} \Phi_{n}$ in the scalar field action, which is then transformed to $S_{m}\left[g, \Phi_{n}\right]$, and construct the Hamiltonian associated to this transformed action, which is given by the above expressions for the stationary case. This is equivalent to making a canonical transformation in the Hamiltonian formulation of the theory. One then introduces an operator $\hat{\Phi}_{n}[g] \equiv e^{(n-2) \varpi / 2} \hat{\Phi}_{n}[\bar{g}]$, which can be identified as the field operator in the Heisenberg picture in the stationary spacetime $\left(\mathcal{M}, g_{a b}\right)$. The associated Hamiltonian operator can be identified with the operator $\hat{H}_{R}[g]$ constructed above (and, obviously, it coincides with $\hat{E}_{R}[\bar{g}]$ ). Note that this Hamiltonian or energy operator generates the time evolution of the operator $\hat{\Phi}_{n}[g]$ rather than that of the "physical" field $\hat{\Phi}_{n}[\bar{g}]$. A generalization of this last approach has been used in Ref. [75] for scalar fields with arbitrary mass and arbitrary coupling to the curvature in a RW spacetime to construct a time-dependent Hamiltonian operator whose ground state at each fixed instant of time is a Hadamard state. A similar construction starting with the above energy functional is given in Ref. [5]. In the massless conformally coupled case, these time-dependent Hamiltonian constructions reduce to the construction sketched in this appendix.

\section{Wick's theorem for thermal states}

From the Hamiltonian operator (B6) (here, we drop the subindex $R$ ), we can define a state of thermal equilibrium for the scalar field as in Eq. (4.1). Following partially the proof presented in the appendix of Ref. [78], we shall next show how Wick's theorem can be generalized for the associated thermal $N$-point functions. First, note that, from Eq. (B7),

$$
\hat{a}_{k}^{(\alpha)} e^{-\beta \hat{H}}=e^{-\alpha \beta \omega_{k}} e^{-\beta \hat{H}} \hat{a}_{k}^{(\alpha)},
$$

where $\alpha=+,-, \hat{a}_{k}^{(+)} \equiv \hat{a}_{k}$ and $\hat{a}_{k}^{(-)} \equiv \hat{a}_{k}^{\dagger}$. Using this and the cyclic property of the trace, we get

$$
\left\langle\hat{a}_{k}^{(\alpha)} \hat{a}_{l}^{(\gamma)}\right\rangle_{T}=\frac{1}{1-e^{-\alpha \beta \omega_{k}}}\left[\hat{a}_{k}^{(\alpha)}, \hat{a}_{l}^{(\gamma)}\right],
$$

where we have used the commutator $\left[\hat{a}_{k}^{(\alpha)}, \hat{a}_{l}^{(\gamma)}\right]$ to represent either $\delta_{k l},-\delta_{k l}$ or 0 (such commutator does not represent an operator in the last equation). Writing the field operator $\hat{\Phi}_{n}(x)$ in terms of the operators $\hat{a}_{k}^{(\alpha)}$, the associated fourpoint thermal functions can be expressed in terms of $\left\langle\hat{a}_{k}^{(\alpha)} \hat{a}_{l}^{(\gamma)} \hat{a}_{r}^{(\delta)} \hat{a}_{s}^{(\sigma)}\right\rangle_{T}$. Taking into account that the commutator $\left[\hat{a}_{k}^{(\alpha)}, \hat{a}_{l}^{(\gamma)}\right]$ is a c-number, one has the following identity:

$$
\hat{a}_{k}^{(\alpha)} \hat{a}_{l}^{(\gamma)} \hat{a}_{r}^{(\delta)} \hat{a}_{s}^{(\sigma)}=\left[\hat{a}_{k}^{(\alpha)}, \hat{a}_{l}^{(\gamma)}\right] \hat{a}_{r}^{(\delta)} \hat{a}_{s}^{(\sigma)}+\left[\hat{a}_{k}^{(\alpha)}, \hat{a}_{r}^{(\delta)}\right] \hat{a}_{l}^{(\gamma)} \hat{a}_{s}^{(\sigma)}+\left[\hat{a}_{k}^{(\alpha)}, \hat{a}_{s}^{(\sigma)}\right] \hat{a}_{l}^{(\gamma)} \hat{a}_{r}^{(\delta)}+\hat{a}_{l}^{(\gamma)} \hat{a}_{r}^{(\delta)} \hat{a}_{s}^{(\sigma)} \hat{a}_{k}^{(\alpha)} .
$$

On the other hand, from Eq. (B9) and the cyclic property of the trace, we have 


$$
\left.\left\langle\hat{a}_{l}^{(\gamma)} \hat{a}_{r}^{(\delta)} \hat{a}_{s}^{(\sigma)} \hat{a}_{k}^{(\alpha)}\right\rangle_{T}=e^{-\alpha \beta \omega_{k}\left\langle\hat{a}_{k}^{(\alpha)}\right.} \hat{a}_{l}^{(\gamma)} \hat{a}_{r}^{(\delta)} \hat{a}_{s}^{(\sigma)}\right\rangle_{T} .
$$

Using the last two equations, we get

$$
\left\langle\hat{a}_{k}^{(\alpha)} \hat{a}_{l}^{(\gamma)} \hat{a}_{r}^{(\delta)} \hat{a}_{s}^{(\sigma)}\right\rangle_{T}=\frac{1}{\left(1-e^{\left.-\alpha \beta \omega_{k}\right)}\right.}\left\{\left[\hat{a}_{k}^{(\alpha)}, \hat{a}_{l}^{(\gamma)}\right]\left\langle\hat{a}_{r}^{(\delta)} \hat{a}_{s}^{(\sigma)}\right\rangle_{T}+\left[\hat{a}_{k}^{(\alpha)}, \hat{a}_{r}^{(\delta)}\right]\left\langle\hat{a}_{l}^{(\gamma)} \hat{a}_{s}^{(\sigma)}\right\rangle_{T}+\left[\hat{a}_{k}^{(\alpha)}, \hat{a}_{s}^{(\sigma)}\right]\left\langle\hat{a}_{l}^{(\gamma)} \hat{a}_{r}^{(\delta)}\right\rangle_{T}\right\},
$$

which, from Eq. (B10), yields

$$
\left\langle\hat{a}_{k}^{(\alpha)} \hat{a}_{l}^{(\gamma)} \hat{a}_{r}^{(\delta)} \hat{a}_{s}^{(\sigma)}\right\rangle_{T}=\left\langle\hat{a}_{k}^{(\alpha)} \hat{a}_{l}^{(\gamma)}\right\rangle_{T}\left\langle\hat{a}_{r}^{(\delta)} \hat{a}_{s}^{(\sigma)}\right\rangle_{T}+\left\langle\hat{a}_{k}^{(\alpha)} \hat{a}_{r}^{(\delta)}\right\rangle_{T}\left\langle\hat{a}_{l}^{(\gamma)} \hat{a}_{s}^{(\sigma)}\right\rangle_{T}+\left\langle\hat{a}_{k}^{(\alpha)} \hat{a}_{s}^{(\sigma)}\right\rangle_{T}\left\langle\hat{a}_{l}^{(\gamma)} \hat{a}_{r}^{(\delta)}\right\rangle_{T},
$$

and, hence, we have

$$
\begin{aligned}
\left\langle\hat{\Phi}_{n}\left(x_{1}\right) \hat{\Phi}_{n}\left(x_{2}\right) \hat{\Phi}_{n}\left(x_{3}\right) \hat{\Phi}_{n}\left(x_{4}\right)\right\rangle_{T}= & \left\langle\hat{\Phi}_{n}\left(x_{1}\right) \hat{\Phi}_{n}\left(x_{2}\right)\right\rangle_{T}\left\langle\hat{\Phi}_{n}\left(x_{3}\right) \hat{\Phi}_{n}\left(x_{4}\right)\right\rangle_{T}+\left\langle\hat{\Phi}_{n}\left(x_{1}\right) \hat{\Phi}_{n}\left(x_{3}\right)\right\rangle_{T}\left\langle\hat{\Phi}_{n}\left(x_{2}\right) \hat{\Phi}_{n}\left(x_{4}\right)\right\rangle_{T} \\
& +\left\langle\hat{\Phi}_{n}\left(x_{1}\right) \hat{\Phi}_{n}\left(x_{4}\right)\right\rangle_{T}\left\langle\hat{\Phi}_{n}\left(x_{2}\right) \hat{\Phi}_{n}\left(x_{3}\right)\right\rangle_{T} .
\end{aligned}
$$

A similar expression holds for the four-point function of time-ordered products (consider $t_{1}>t_{2}>t_{3}>t_{4}$ in the last equation). These results can be easily generalized to thermal $2 N$-point functions $(N \in \mathbb{N})$. On the other hand, from Eq. (B9), we can see that $\left\langle\hat{a}_{k}^{(\alpha)}\right\rangle_{T}=0$ and, following similar steps, we can show that the thermal $(2 N-1)$-point functions vanish.

[1] R. M. Wald, Quantum Field Theory in Curved Spacetime and Black Hole Thermodynamics (The University of Chicago Press, Chicago, 1994).

[2] R. M. Wald, Commun. Math. Phys. 54, 1 (1977).

[3] S. A. Fulling, Aspects of Quantum Field Theory in Curved Space-time (Cambridge University Press, Cambridge, England, 1989).

[4] N. D. Birrell and P. C. W. Davies, Quantum Fields in Curved Space (Cambridge University Press, Cambridge, England, 1982).

[5] A. A. Grib, S. G. Mamayev, and V. M. Mostepanenko, Vacuum Quantum Effects in Strong Fields (Friedmann Laboratory Publishing, St. Petersburg, 1994).

[6] É. É. Flanagan and R. M. Wald, Phys. Rev. D 54, 6233 (1996); M. Visser, Phys. Lett. B 415, 8 (1997); gr-qc/9710020.

[7] L. H. Ford, Ann. Phys. (N.Y.) 144, 238 (1982).

[8] C.-I. Kuo and L. H. Ford, Phys. Rev. D 47, 4510 (1993); N. G. Phillips and B.-L. Hu, ibid. 55, 6123 (1997).

[9] B.-L. Hu, Physica A 158, 399 (1989).

[10] R. P. Feynman and F. L. Vernon, Ann. Phys. (N.Y.) 24, 118 (1963).

[11] R. P. Feynman and A. R. Hibbs, Quantum Mechanics and Path Integrals (McGraw-Hill, New York, 1965).

[12] E. Calzetta and B.-L. Hu, Phys. Rev. D 49, 6636 (1994).

[13] B.-L. Hu and A. Matacz, Phys. Rev. D 51, 1577 (1995).

[14] B.-L. Hu and S. Sinha, Phys. Rev. D 51, 1587 (1995).

[15] A. O. Caldeira and A. J. Legget, Physica A 121, 587 (1983).

[16] B.-L. Hu, J. P. Paz, and Y. Zhang, Phys. Rev. D 45, 2843 (1992).

[17] B.-L. Hu and A. Matacz, Phys. Rev. D 49, 6612 (1994).

[18] C. Greiner and B. Müller, Phys. Rev. D 55, 1026 (1997).

[19] A. Campos and E. Verdaguer, Phys. Rev. D 53, 1927 (1996).

[20] F. C. Lombardo and F. D. Mazzitelli, Phys. Rev. D 55, 3889 (1997).
[21] A. Campos and E. Verdaguer, Int. J. Theor. Phys. 36, 2525 (1997).

[22] E. Calzetta, A. Campos, and E. Verdaguer, Phys. Rev. D 56, 2163 (1997).

[23] A. Campos and B.-L. Hu, Phys. Rev. D 58, 125021 (1998).

[24] A. Campos and B.-L. Hu, gr-qc/9819234.

[25] E. Calzetta and E. Verdaguer, Phys. Rev. D 59, 083513 (1999).

[26] B.-L. Hu, J. P. Paz, and Y. Zhang, Phys. Rev. D 47, 1576 (1993).

[27] A. Matacz, Phys. Rev. D 55, 1860 (1997).

[28] M. Morikawa, Phys. Rev. D 33, 3607 (1986); D.-S. Lee and D. Boyanovsky, Nucl. Phys. B406, 631 (1993).

[29] R. Zh. Shaisultanov, hep-th/9509154; hep-th/9512144.

[30] M. Gleiser and R. O. Ramos, Phys. Rev. D 50, 2441 (1994); D. Boyanovsky, H. J. de Vega, R. Holman, D. S. Lee, and A. Singh, ibid. 51, 4419 (1995); E. Calzetta and B.-L. Hu, ibid. 55, 3536 (1997); M. Yamaguchi and J. Yokoyama, ibid. 56, 4544 (1997); S. A. Ramsey, B.-L. Hu, and A. M. Stylianopoulos, ibid. 57, 6003 (1998).

[31] R. Martin and E. Verdaguer, gr-qc/9811070.

[32] M. Gell-Mann and J. B. Hartle, Phys. Rev. D 47, 3345 (1993).

[33] J. B. Hartle, in Gravitation and Quantizations, Proceedings of the1992 Les Houches Summer School, edited by B. Julia and J. Zinn-Justin (North-Holland, Amsterdam, 1995), gr-qc/9304006, and references therein.

[34] H. F. Dowker and J. J. Halliwell, Phys. Rev. D 46, 1580 (1992).

[35] J. J. Halliwell, Phys. Rev. D 48, 4785 (1993); 57, 2337 (1998).

[36] J. T. Whelan, Phys. Rev. D 57, 768 (1998); gr-qc/9702003.

[37] R. Martin and E. Verdaguer, gr-qc/9812063.

[38] H. B. Callen and T. A. Welton, Phys. Rev. 83, 34 (1951); W. Bernard and H. B. Callen, Rev. Mod. Phys. 31, 1017 (1959); L. D. Landau, E. M. Lifshitz, and L. P. Pitaevskii, Course of 
Theoretical Physics, Vol. 5, 3rd ed.: Statistical Physics, Part 1 (Pergamon, London, 1980); G. Parisi, Statistical Field Theory (Frontiers in Physics Series, Addison-Wesley, Reading, MA, 1988).

[39] R. Kubo, J. Phys. Soc. Jpn. 12, 570 (1957); in Lectures in Theoretical Physics, Vol. I, Proceedings of the 1958 Summer Institute for Theoretical Physics, University of Colorado, Boulder, Colorado, edited by W. E. Brittin and L. G. Dunham (Interscience, New York, 1959); Rep. Prog. Phys. 29, 255 (1966).

[40] H. Grabert, P. Schramm, and G.-L. Ingold, Phys. Rep. 168, 115 (1988).

[41] J. Schwinger, J. Math. Phys. 2, 407 (1961).

[42] J. Weber, Phys. Rev. 101, 1620 (1956).

[43] R. Kubo, M. Toda, and N. Hashitsume, Statistical Physics II (Springer-Verlag, Berlin, 1985).

[44] P. C. Martin and J. Schwinger, Phys. Rev. 115, 1342 (1959); L. P. Kadanoff and P. C. Martin, Ann. Phys. (N.Y.) 24, 419 (1963); M. Plischke and B. Bergersen, Equilibrium Statistical Physics, 2nd ed. (World Scientific, Singapore, 1994).

[45] R. Jackiw and V. P. Nair, Phys. Rev. D 48, 4991 (1993).

[46] P. Candelas and D. W. Sciama, Phys. Rev. Lett. 38, 1372 (1977); D. W. Sciama, P. Candelas, and D. Deutsch, Adv. Phys. 30, 327 (1981); D. W. Sciama, in Quantum Gravity 2: A Second Oxford Symposium, edited by C. J. Isham, R. Penrose, and D. W. Sciama (Clarendon Press, Oxford, 1981).

[47] E. Mottola, Phys. Rev. D 33, 2136 (1986).

[48] B.-L. Hu, gr-qc/9902064.

[49] B.-L. Hu, A. Raval, and S. Sinha, in Black Holes, Gravitational Radiation and the Universe, edited by B. R. Iyer and B. Bhawal (Kluwer Academic Publishers, Dordrecht, 1999).

[50] R. M. Wald, General Relativity (The University of Chicago Press, Chicago, 1984).

[51] S. M. Christensen, Phys. Rev. D 14, 2490 (1976); 17, 946 (1978)

[52] T. S. Bunch, J. Phys. A 12, 517 (1979).

[53] S. A. Fulling and L. Parker, Ann. Phys. (N.Y.) 87, 176 (1974).

[54] J. Schwinger, Phys. Rev. 128, 2425 (1962); P. M. Bakshi and K. T. Mahanthappa, J. Math. Phys. 4, 1 (1963); 4, 12 (1963); L. V. Keldysh, Zh. Éksp. Teor. Fiz. 47, 1515 (1964) [Sov. Phys. JETP 20, 1018 (1965)]; J. Schwinger, Particles, Sources, and Fields (Addison-Wesley, Reading, MA, 1970).

[55] K.-C. Chou, Z.-B. Su, B.-L. Hao, and L. Yu, Phys. Rep. 118, 1 (1985); N. P. Landsman and Ch. G. van Weert, ibid. 145, 141 (1987); E. Calzetta and B.-L. Hu, Phys. Rev. D 35, 495 (1987).

[56] A. Campos and E. Verdaguer, Phys. Rev. D 49, 1861 (1994).

[57] J. F. Donoghue, Phys. Rev. Lett. 72, 2996 (1994); Phys. Rev. D 50, 3874 (1994); Helv. Phys. Acta 69, 269 (1996); in Ad- vanced School on Effective Theories, edited by F. Cornet and M. J. Herrero (World Scientific, Singapore, 1996), gr-qc/9512024; gr-qc/9712070.

[58] S. Weinberg, The Quantum Theory of Fields, Vols. I and II (Cambridge University Press, Cambridge, England, 1995 and 1996).

[59] R. M. Wald, Commun. Math. Phys. 70, 221 (1979); T. S. Bunch, Ann. Phys. (N.Y.) 131, 118 (1981); M. Lüscher, ibid. 142, 359 (1982).

[60] B. S. Kay, Commun. Math. Phys. 62, 55 (1978).

[61] J. S. Dowker and R. Critchley, Phys. Rev. D 15, 1484 (1977); J. S. Dowker and G. Kennedy, J. Phys. A 11, 895 (1978); G. Kennedy, R. Critchley, and J. S. Dowker, Ann. Phys. (N.Y.) 125, 346 (1980).

[62] G. Gibbons and M. J. Perry, Proc. R. Soc. London A358, 467 (1978).

[63] I. R. Senitzky, Phys. Rev. 119, 670 (1960); P. Ullersma, Physica (Amsterdam) 32, 27 (1966); ibid. 32, 90 (1966); K. Lindenberg and B. J. West, Phys. Rev. A 30, 568 (1984).

[64] G. W. Ford, M. Kac, and P. Mazur, J. Math. Phys. 6, 504 (1965); H. Mori, Prog. Theor. Phys. 33, 423 (1965); H. Dekker, Phys. Rep. 80, 1 (1981); K. Lindenberg and B. J. West, The Nonequilibrium Statistical Mechanics of Open and Closed Systems (VCH Publishers, New York, 1990).

[65] L. Schwartz, Théorie des distributions, Tomes I et II (Hermann, Paris, 1957 and 1959); A. H. Zemanian, Distribution Theory and Transform Analysis (Dover, New York, 1987).

[66] H. Nyquist, Phys. Rev. 32, 110 (1928); M. S. Green, J. Chem. Phys. 19, 1036 (1951); H. B. Callen and R. F. Greene, Phys. Rev. 86, 702 (1952).

[67] R. Zwanzig, J. Stat. Phys. 9, 215 (1973).

[68] W. Israel, in General Relativity, papers in honor of J. L. Synge, edited by L. O'Raifeartaigh (Oxford University Press, London, 1972).

[69] J. H. Cooke, Am. J. Phys. 45, 1168 (1977); G. Kennedy, J. Phys. A 11, L77 (1978); B.-L. Hu, Phys. Lett. 108B, 19 (1982); P. Amsterdamski, Phys. Rev. D 35, 1209 (1987); J. P. Paz, ibid. 41, 1054 (1990).

[70] C. R. Cramer and B. S. Kay, Phys. Rev. D 57, 1052 (1998).

[71] J. Céspedes and E. Verdaguer, Phys. Rev. D 41, 1022 (1990).

[72] R. U. Sexl and H. K. Urbantke, Phys. Rev. 179, 1247 (1969).

[73] R. Martín and E. Verdaguer, in preparation.

[74] J. A. Frieman, Phys. Rev. D 39, 389 (1989).

[75] N. Weiss, Phys. Rev. D 34, 1768 (1986).

[76] B. S. DeWitt, Phys. Rep., Phys. Lett. 19C, 297 (1975).

[77] L. H. Ford, Phys. Rev. D 11, 3370 (1975).

[78] M. Le Bellac, Thermal Field Theory (Cambridge University Press, Cambridge, England, 1996). 